\title{
Existence and stability of bushes of vibrational modes for octahedral mechanical systems with Lennard-Jones potential
}

\author{
G.M. Chechin, A.V. Gnezdilov and M.Yu. Zekhtser \\ Rostov State University, Rostov-on-Don, Russia
}

\begin{abstract}
The special nonlinear dynamical regimes, "bushes of normal modes", can exist in the $N$-particle Hamiltonian systems with discrete symmetry [Physica D 117 (1998) 43]. The dimension of the bush can be essentially less than that of the whole mechanical system. One-dimensional bushes represent the similar nonlinear normal modes introduced by Rosenberg. A given bush can be excited by imposing the appropriate initial conditions, and the energy of the initial excitation turns out to be trapped in this bush.

In the present paper, we consider all possible vibrational bushes in the simple octahedral mechanical system and discuss their stability under assumption that the interactions between particles are described by the Lennard-Jones potential.
\end{abstract}

\section{Introduction}

A new concept, "bushes of normal modes", was introduced for nonlinear mechanical systems with discrete symmetry in [1, 2] A given bush represents a certain superposition of the modes associated with different irreducible representations (irreps) of the symmetry group $G$ of the mechanical system in equilibrium. The coefficients of this superposition are time-dependent functions for which the exact ordinary differential equations can be obtained. In this sense, the bush can be considered as a dynamical object whose dimensionality is generally less than that of the original mechanical system. The following propositions were justified in previous papers [1, 2, 3]:

1. A certain subgroup $G_{D}$ of the symmetry group $G$ corresponds to a given bush, and this bush can be excited by imposing the appropriate initial conditions with the above symmetry group $G_{D} \subset G$.

2. Each mode belonging to the bush possesses its own symmetry group which is greater than or equal to the group $G_{D}$ of the whole bush.

3. In spite of evolving mode amplitudes, the complete collection of modes in the given bush is preserved in time and, in this sense, the bush can be considered as a geometrical object.

4. The energy of the initial excitation is trapped in the bush, i.e. it cannot spread to the modes which do not belong to the bush, because of the symmetry restrictions. 
5. As an indivisible nonlinear object, the bush exists because of force interactions between the modes contained in it.

6. Taking into account the concrete type of interactions between particles of the considered mechanical system can only reduce the dimension of the given bush.

7. The extension of the bush can be realized as a result of the loss of its stability which is accompanied by spontaneous breaking of the bush symmetry (dynamical analog of phase transition).

The special group-theoretical methods for finding bushes of modes are discussed in [3, 4]. The computer implementation of these methods [5] (see also [6]) allowed us to find bushes of modes for wide classes of mechanical systems with discrete symmetry. In particular, "irreducible" bushes of vibrational modes and symmetry determined similar non-linear normal modes for all N-particle mechanical systems with the symmetry of any of the 230 space groups were found in [4]. The bushes of vibrational modes of small dimensions were found and classified into universality classes for all mechanical systems with point groups of crystallographic symmetry in [7].

Unlike discussions of existence of bushes of modes, a few papers were devoted to the problem of their stability (as an example, we can refer to the investigation of bushes of vibrational modes for FPU- $\alpha$ chains in [8]). Nevertheless, the problem of stability is one of the most important for bush theory. Indeed, we must keep in mind that bushes of modes can be considered as new physical objects only in the case where they are stable in the finite domains of pertinent parameters of the appropriate dynamical systems.

The problem of existence of bushes of modes can be studied with the aid of the grouptheoretical methods only, and appropriate results do not depend on the concrete type of interactions between particles of our mechanical system. In contrast, studying of bush stability depends on these interactions essentially.

In the present paper, after the outline of the bush theory in Sec.2, we consider bushes of vibrational modes for an octahedral mechanical system of mass particles depicted in Fig. 1, with or without a particle in the center of the octahedron. All bushes of modes are presented in Sec.3. The differential equations describing their dynamics are discussed in Sec.4. The stability of the bushes of vibrational modes is investigated in Sec.5 for the case of interactions between particles described by the Lennard-Jones potential.

\section{Bushes of vibrational modes}

A detailed consideration of the theory of bushes of modes with some theorems about their structure and with the appropriate group-theoretical methods can be found in [3]. An extensive description of the computational algorithm for finding bushes in arbitrary crystal structures is presented in [4]. Let us now give an outline of the bush theory with emphasis on the ideas important for our further discussion.

\subsection{Geometrical aspects}

We consider classical Hamiltonian systems of $N$ mass points moving near a single equilibrium state which can be characterized by a certain point or space symmetry group $G$. Let the $3 \times N$-dimensional vector,

$$
\mathbf{X}(t)=\left(\mathbf{x}_{1}(t), \mathbf{x}_{2}(t), \ldots, \mathbf{x}_{N}(t)\right)
$$


describe the displacements $\mathbf{x}_{i}(t)$ of all particles of our mechanical system from their equilibrium positions. (Here we denote by the three-dimensional vector $\mathbf{x}_{i}$ the displacement of the $i$-th particle along the $X, Y$ and $Z$ axes).

The vector $\mathbf{X}(t)$ can be written as a superposition of all basis vectors $\varphi_{i}^{(j)}$ of the irreducible representations $\Gamma_{j}$ of the above mentioned symmetry group $G$ :

$$
\mathbf{X}(t)=\sum_{j, i} \mu_{i}^{(j)}(t) \boldsymbol{\varphi}_{i}^{(j)}
$$

The coefficients $\mu_{i}^{(j)}(t)$ of this superposition depend on time $t$, while the $3 \times N$-dimensional time-independent vectors $\varphi_{i}^{(j)}$ determine the specific patterns of displacements of all particles of our mechanical system.

Note that the basis vectors $\varphi_{i}^{(j)}$ are often called "symmetry-adapted coordinates". In particular, they can be normal coordinates. Hereafter, the term, "mode", means an arbitrary superposition of basis vectors corresponding to a given irrep $\Gamma_{j}$. As a result of this definition, every term $\mu_{i}^{(j)}(t) \boldsymbol{\varphi}_{i}^{(j)}$ in the right-hand side of Eq.(2) is also a mode of the irrep $\Gamma_{j}$. Sometimes, for brevity, we will refer to $\mu_{i}^{(j)}(t)$ as a mode, but a reader must imagine that this time-dependent coefficient is multiplied by the appropriate $3 \times N$-dimensional vector $\boldsymbol{\varphi}_{i}^{(j)}$ to give the mode in the exact sense. We can also speak about vibrational modes because only such type of symmetry-adapted (normal) modes are considered in the present paper.

Every dynamical regime of the considered mechanical system can be described by the appropriate time-dependent vector $\mathbf{X}(t)$ which determines a definite instantaneous configuration of the system. On the other hand, each instantaneous configuration possesses a certain symmetry group $G_{D}$ (in particular, this group may be trivial: $G_{D}=1$ ) which is a subgroup of the symmetry group $G$ of the system in equilibrium $\left(G_{D} \subseteq G\right)$. Moreover, we can also ascribe a certain symmetry group to each basis vector $\varphi_{i}^{(j)}$ and to each mode corresponding to a given irrep $\Gamma_{j}$ (remember that a mode is a superposition of such vectors!), because the definite instantaneous configurations correspond to them. The group $G_{D}$ contains all symmetry elements of group $G$ whose action does not change this configuration.

Let us introduce, as it is usual in group theory, the operators $\hat{g}$ associated with elements $g$ of group $G(g \in G)$ which act on $3 \times N$-dimensional vectors $\mathbf{X}(t)$. I All elements $g \in G$ for which

$$
\hat{g} \mathbf{X}(t)=\mathbf{X}(t)
$$

form a certain subgroup $G_{D} \subseteq G$, and a complete set of the above operators $\hat{g}\left(\forall g \in G_{D}\right)$ represents the group $\widehat{G}_{D}$.

It can be shown that the symmetry group $G_{D}$ is preserved in time in the sense that its elements cannot disappear during time evolution. Actually, this property is a consequence of the principle of determinism in classical mechanics. $\left(g \in G_{D}\right)$, or, formally,

$$
\widehat{G}_{D} \mathbf{X}(t)=\mathbf{X}(t)
$$

is valid for every time $t$. As a consequence, we can classify the different dynamical regimes in our nonlinear dynamical system, described by the vectors $\mathbf{X}(t)$ from Eqs.(1),21), with the aid of symmetry groups corresponding to them.

\footnotetext{
${ }^{1}$ The symmetry elements $g \in G$ act on the vectors of three-dimensional Euclidean space.

${ }^{2}$ The phenomenon of spontaneous breaking of symmetry of a given dynamical regime will be considered in the next section.
} 
Using Eq.(㺼), we can obtain the similar invariance conditions for each individual irrep $\Gamma_{j}$ of the group $G$ (see details in [3]):

$$
\left(\Gamma_{j} \downarrow G_{D}\right) \boldsymbol{\mu}_{j}=\boldsymbol{\mu}_{j}
$$

Here $\left(\Gamma_{j} \downarrow G_{D}\right)$ is a restriction of the irrep $\Gamma_{j}$ to the subgroup $G_{D}$ of the group $G$, and $\boldsymbol{\mu}_{j}=\left(\mu_{1}^{(j)}, \ldots, \mu_{n_{j}}^{(j)}\right)$ is an invariant vector of $\Gamma_{j}\left(n_{j}\right.$ is the dimension of this irrep).

To find all modes contributing to a given dynamical regime with symmetry group $G_{D}$, i.e., for the vector $\mathbf{X}(t)$ from Eq.(2), we must solve linear algebraic equations (5) for each irrep $\Gamma_{j}$ of the group $G$. As a result of this procedure, the invariant vector $\boldsymbol{\mu}_{j}$ for some irreps $\Gamma_{j}$ can turn out to be equal to zero. Such irreps do not contribute to the considered dynamical regime. On the other hand, some nonzero invariant vectors $\boldsymbol{\mu}_{j}$ for multidimensional irreps may be of a very specific form because of definite relations between their components (for example, certain components can be equal to each other, or differ only by sign). The contributions to $\mathbf{X}(t)$ from such irreps $\Gamma_{j}$ possess special forms. In Tables 1, 2, we find, for instance, dynamical regimes for which the contributions from the irrep $\Gamma_{10}$ of the group $O_{h}$ are of the forms $\mu_{1}^{10}(t)\left[\boldsymbol{\varphi}_{1}^{(10)}-\boldsymbol{\varphi}_{2}^{(10)}\right]$ and $\mu_{1}^{10}(t)\left[\boldsymbol{\varphi}_{1}^{(10)}+\boldsymbol{\varphi}_{2}^{(10)}+\boldsymbol{\varphi}_{3}^{(10)}\right]$.

Table 1: Bushes of vibrational modes and their stability domains for the octahedral structure with Lennard-Jones potential

\begin{tabular}{|c|c|c|c|c|c|}
\hline N & $\begin{array}{c}\text { Point } \\
\text { group }\end{array}$ & Generators & Dim. & Bush of modes & $\begin{array}{c}\text { Range of bush } \\
\text { stability }\end{array}$ \\
\hline 1 & $O_{h}$ & $13,14,33$ & 1 & $\underline{\mu_{1}^{(1)} \boldsymbol{\varphi}_{1}^{(1)}}$ & $R=0.001$ \\
\hline 2 & $D_{4 h}$ & $2,14,25$ & 2 & $\mu_{1}^{(1)} \boldsymbol{\varphi}_{1}^{(1)}+\mu_{1}^{(5)} \boldsymbol{\varphi}_{1}^{(5)}$ & $R=0.009$ \\
\hline 3 & $D_{2 d}^{\prime}$ & 13,38 & 3 & $\mu_{1}^{(1)} \boldsymbol{\varphi}_{1}^{(1)}+\mu_{1}^{(5)} \boldsymbol{\varphi}_{1}^{(5)}+\mu_{3}^{(8)} \boldsymbol{\varphi}_{3}^{(8)}$ & $R=0.028$ \\
\hline 4 & $C_{4 v}$ & 14,37 & 3 & $\mu_{1}^{(1)} \boldsymbol{\varphi}_{1}^{(1)}+\mu_{1}^{(5)} \boldsymbol{\varphi}_{1}^{(5)}+\mu_{3}^{(10)} \boldsymbol{\varphi}_{3}^{(10)}$ & $R=0.003$ \\
\hline 5 & $D_{2 h}^{\prime}$ & $4,13,25$ & 3 & $\mu_{1}^{(1)} \boldsymbol{\varphi}_{1}^{(1)}+\mu_{1}^{(5)} \boldsymbol{\varphi}_{1}^{(5)}+\mu_{3}^{(7)} \boldsymbol{\varphi}_{3}^{(7)}$ & $R=0.055$ \\
\hline 6 & $C_{2 v}^{\prime}$ & 13,28 & 5 & $\mu_{1}^{(1)} \boldsymbol{\varphi}_{1}^{(1)}+\mu_{1}^{(5)} \boldsymbol{\varphi}_{1}^{(5)}+\mu_{3}^{(7)} \boldsymbol{\varphi}_{3}^{(7)}+$ & $R_{1}=0.001$ \\
& & & & $\frac{\mu_{1}^{(8)}\left(\boldsymbol{\varphi}_{1}^{(8)}+\boldsymbol{\varphi}_{2}^{(8)}\right)+\mu_{1}^{(10)}\left(\boldsymbol{\varphi}_{1}^{(10)}-\boldsymbol{\varphi}_{2}^{(10)}\right)}{R_{2}=0.002}$ \\
\hline 7 & $D_{3}$ & 9,13 & 3 & $\mu_{1}^{(1)} \boldsymbol{\varphi}_{1}^{(1)}+\mu_{1}^{(7)}\left(\boldsymbol{\varphi}_{1}^{(7)}+\boldsymbol{\varphi}_{2}^{(7)}+\boldsymbol{\varphi}_{3}^{(7)}\right)+$ & $R=0.002$ \\
\hline 8 & $C_{3 v}$ & 9,37 & 3 & $\frac{\mu_{1}^{(8)}\left(\boldsymbol{\varphi}_{1}^{(8)}+\boldsymbol{\varphi}_{2}^{(8)}+\boldsymbol{\varphi}_{3}^{(8)}\right)}{\boldsymbol{\varphi}_{1}^{(1)}+\mu_{1}^{(7)}\left(\boldsymbol{\varphi}_{1}^{(7)}+\boldsymbol{\varphi}_{2}^{(7)}+\boldsymbol{\varphi}_{3}^{(7)}\right)+}$ & $R=0.025$ \\
\hline 9 & $D_{3 d}$ & 13,33 & 2 & $\frac{\mu_{1}^{(10)}\left(\boldsymbol{\varphi}_{1}^{(10)}+\boldsymbol{\varphi}_{2}^{(10)}+\boldsymbol{\varphi}_{3}^{(10)}\right)}{\boldsymbol{\varphi}_{1}^{(1)}+\mu_{1}^{(7)} \underline{\left(\boldsymbol{\varphi}_{1}^{(7)}+\boldsymbol{\varphi}_{2}^{(7)}+\boldsymbol{\varphi}_{3}^{(7)}\right)}}$ & $R=0.078$ \\
\hline
\end{tabular}

Actually, Eq.(5) can be considered as a source of certain selection rules for spreading excitation from the root mode to a number of other (secondary) modes. Indeed, if a certain mode with the symmetry group $G_{D}$ is excited at the initial instant (we call it the "root" mode), this group determines the symmetry of the whole bush. The condition that the appropriate dynamical regime $\mathbf{X}(t)$ must be invariant under the action of the above group 
Table 2: Bushes of vibrational modes and their stability domains for the octahedral structure with Lennard-Jones potential

\begin{tabular}{|c|c|c|c|c|c|}
\hline $\mathrm{N}$ & $\begin{array}{l}\text { Point } \\
\text { group }\end{array}$ & Generators & Dim. & Bush of modes & $\begin{array}{l}\text { Range of bush } \\
\text { stability }\end{array}$ \\
\hline 10 & $D_{2 h}$ & $2,4,25$ & 3 & $\mu_{1}^{(1)} \boldsymbol{\varphi}_{1}^{(1)}+\mu_{1}^{(5)} \boldsymbol{\varphi}_{1}^{(5)}+\mu_{2}^{(5)} \boldsymbol{\varphi}_{2}^{(5)}$ & $R^{\prime}=0.006$ \\
\hline 11 & $D_{2}^{\prime}$ & 4,13 & 4 & $\mu_{1}^{(1)} \boldsymbol{\varphi}_{1}^{(1)}+\mu_{1}^{(5)} \boldsymbol{\varphi}_{1}^{(5)}+\mu_{3}^{(7)} \boldsymbol{\varphi}_{3}^{(7)}+\mu_{3}^{(8)} \boldsymbol{\varphi}_{3}^{(8)}$ & $R^{\prime}=0.027$ \\
\hline 12 & $C_{2 v}$ & 4,26 & 5 & $\begin{array}{c}\mu_{1}^{(1)} \boldsymbol{\varphi}_{1}^{(1)}+\mu_{1}^{(5)} \boldsymbol{\varphi}_{1}^{\left(\overline{5)}+\mu_{2}^{(5)} \boldsymbol{\varphi}_{2}^{(5)}+\right.} \\
\mu_{3}^{(8)} \boldsymbol{\varphi}_{3}^{(8)}+\mu_{3}^{(10)} \boldsymbol{\varphi}_{3}^{(10)}\end{array}$ & $R^{\prime}=0.001$ \\
\hline 13 & $C_{2 v}^{\prime \prime}$ & 4,37 & 4 & $\mu_{1}^{(1)} \boldsymbol{\varphi}_{1}^{(1)}+\mu_{1}^{(5)} \boldsymbol{\varphi}_{1}^{(5)}+\underline{\mu}_{3}^{(7)} \boldsymbol{\varphi}_{3}^{(7)}+\mu_{3}^{(10)} \boldsymbol{\varphi}_{3}^{(10)}$ & $R^{\prime}=0.003$ \\
\hline 14 & $C_{2}^{\prime}$ & 13 & 7 & $\begin{array}{c}\mu_{1}^{(1)} \boldsymbol{\varphi}_{1}^{(1)}+\mu_{1}^{(5)} \boldsymbol{\varphi}_{1}^{(5)}+\overline{\mu_{1}^{(7)}\left(\boldsymbol{\varphi}_{1}^{(7)}+\boldsymbol{\varphi}_{2}^{(7)}\right)+} \\
\mu_{3}^{(7)} \boldsymbol{\varphi}_{3}^{(7)}+\mu_{1}^{(8)}\left(\boldsymbol{\varphi}_{1}^{(8)}+\boldsymbol{\varphi}_{2}^{(8)}\right)+ \\
\underline{\mu_{3}^{(8)} \boldsymbol{\varphi}_{3}^{(8)}}+\overline{\mu_{1}^{(10)}\left(\boldsymbol{\varphi}_{1}^{(10)}-\boldsymbol{\varphi}_{2}^{(10)}\right)}\end{array}$ & $R^{\prime}=0.001$ \\
\hline 15 & $C_{s}$ & 28 & 8 & $\begin{array}{c}\mu_{1}^{(1)} \boldsymbol{\varphi}_{1}^{(1)}+\mu_{1}^{(5)} \boldsymbol{\varphi}_{1}^{(5)}+\mu_{2}^{(5)} \boldsymbol{\varphi}_{2}^{(5)}+\mu_{3}^{(7)} \boldsymbol{\varphi}_{3}^{(7)}+ \\
\mu_{1}^{(8)} \boldsymbol{\varphi}_{1}^{(8)}+\mu_{2}^{(8)} \boldsymbol{\varphi}_{2}^{(8)}+\mu_{1}^{(10)} \boldsymbol{\varphi}_{1}^{(10)}+\mu_{2}^{(10)} \boldsymbol{\varphi}_{2}^{(10)} \\
\end{array}$ & $\begin{array}{l}R_{1}^{\prime}=0.013 \\
R_{2}^{\prime}=0.002\end{array}$ \\
\hline 16 & $C_{s}^{\prime}$ & 37 & 7 & $\begin{array}{c}\mu_{1}^{(1)} \boldsymbol{\varphi}_{1}^{(1)}+\mu_{1}^{(5)} \boldsymbol{\varphi}_{1}^{(5)}+\mu_{1}^{(7)}\left(\boldsymbol{\varphi}_{1}^{(7)}+\boldsymbol{\varphi}_{2}^{(7)}\right)+ \\
\mu_{3}^{(7)} \boldsymbol{\varphi}_{3}^{(7)}+\mu_{1}^{(8)}\left(\boldsymbol{\varphi}_{1}^{(8)}-\boldsymbol{\varphi}_{2}^{(8)}\right)+ \\
\mu_{1}^{(10)}\left(\boldsymbol{\varphi}_{1}^{(10)}+\boldsymbol{\varphi}_{2}^{(10)}\right)+\mu_{3}^{(10)} \boldsymbol{\varphi}_{3}^{(10)} \\
\end{array}$ & $R^{\prime}=0.007$ \\
\hline 17 & $C_{3}$ & 9 & 4 & $\begin{array}{c}\mu_{1}^{(1)} \boldsymbol{\varphi}_{1}^{(1)}+\mu_{1}^{(7)}\left(\boldsymbol{\varphi}_{1}^{(7)}+\boldsymbol{\varphi}_{2}^{(7)}+\boldsymbol{\varphi}_{3}^{(7)}\right)+ \\
\frac{\mu_{1}^{(8)}\left(\boldsymbol{\varphi}_{1}^{(8)}+\boldsymbol{\varphi}_{2}^{(8)}+\boldsymbol{\varphi}_{3}^{(8)}\right)+}{\mu_{1}^{(10)}\left(\boldsymbol{\varphi}_{1}^{(10)}+\boldsymbol{\varphi}_{2}^{(10)}+\boldsymbol{\varphi}_{3}^{(10)}\right)}\end{array}$ & $R^{\prime}=0.005$ \\
\hline 18 & $C_{i}$ & 25 & 6 & $\begin{array}{c}\mu_{1}^{(1)} \boldsymbol{\varphi}_{1}^{(1)}+\mu_{1}^{(5)} \boldsymbol{\varphi}_{1}^{(5)}+\mu_{2}^{(5)} \boldsymbol{\varphi}_{2}^{(5)}+ \\
\mu_{1}^{(7)} \boldsymbol{\varphi}_{1}^{(7)}+\mu_{2}^{(7)} \boldsymbol{\varphi}_{2}^{(7)}+\mu_{3}^{(7)} \boldsymbol{\varphi}_{3}^{(7)}\end{array}$ & $R^{\prime \prime}=0.001$ \\
\hline
\end{tabular}

$G_{D}$ leads to Eq.(勾) and then to Eq.(5). If the vector $\boldsymbol{\mu}_{j}$ for a given irrep $\Gamma_{j}$ proves to be a zero vector, then there are no modes belonging to this irrep which contribute to $\mathbf{X}(t)$, i.e., the initial excitation cannot spread from the root mode to the secondary modes associated with the irrep $\Gamma_{j}$.

Note that basis vectors associated with a given irrep $\Gamma_{j}$ in Eq.(2) turn out to be equal to zero when this irrep is not contained in the decomposition of the full vibrational irrep $\Gamma$ into its irreducible parts $\Gamma_{j}$. This is a source of the additional selection rules which reduce the number of possible vibrational modes in the considered bush. Trying every irrep $\Gamma_{j}$ in Eq.(5) and analyzing the above mentioned decomposition of the vibrational representation $\Gamma$, we obtain the whole bush of modes with the symmetry group $G_{D}$ in the explicit form.

Actually, we have just outlined the group-theoretical scheme for finding bushes of vibrational modes for arbitrary mechanical systems. The detailed consideration of this scheme can be found in [3, 4].

In conclusion, let us rewrite the vector $\mathbf{X}(t)$ from Eq.(2) as the sum of contributions $\boldsymbol{\Delta}_{j}$ 
from all different irreps $\Gamma_{j}$ of the group $G$. For each irrep $\Gamma_{j}$ with the dimension $n_{j}$, we introduce a "supervector"

$$
\boldsymbol{\Phi}_{j}=\left\{\boldsymbol{\varphi}_{1}^{(j)}, \ldots, \boldsymbol{\varphi}_{n_{j}}^{(j)}\right\}
$$

which represents the complete set of basis vectors $\boldsymbol{\varphi}_{i}^{(j)}\left(i=1, \ldots, n_{j}\right)$ of this irrep. Remembering that the invariant vector $\boldsymbol{\mu}_{j}$ has the form,

$$
\boldsymbol{\mu}_{j}=\left\{\mu_{1}^{(j)}, \ldots, \mu_{n_{j}}^{(j)}\right\},
$$

we can rewrite Eq.(2) in the following compact form:

$$
\mathbf{X}(t)=\sum_{j} \boldsymbol{\Delta}_{j}=\sum_{j}\left(\boldsymbol{\mu}_{j}(t) \mid \boldsymbol{\Phi}_{j}\right) .
$$

Here we introduced a formal "scalar" product

$$
\left(\boldsymbol{\mu}_{j}(t) \mid \boldsymbol{\Phi}_{j}\right)=\sum_{i=1}^{n_{j}} \mu_{i}^{(j)}(t) \boldsymbol{\varphi}_{i}^{(j)}
$$

of $n_{j}$-dimensional vectors $\boldsymbol{\mu}_{j}$ and $\boldsymbol{\Phi}_{j}$ (actually, this product represents a certain $3 \times N$ dimensional vector $\left.\boldsymbol{\Delta}_{j} !\right)$.

\subsection{Dynamical aspects}

Let us return to Eq.(2). We speak about geometrical aspects of the bush theory when concentrating our attention on basis vectors $\boldsymbol{\varphi}_{i}^{(j)}$, and we speak about dynamical aspects of this theory when we focus on time-dependent coefficients $\mu_{i}^{(j)}(t)$, which will be also called "modes" (see comments on this term in Sec.2.1).

If interactions between the particles of our mechanical system are known, exact dynamical equations describing the time evolution of a given bush can be written. We will consider this question in Sec. 1 of the present paper, while some general statements about the theory of bush dynamics are considered here.

Two types of interactions between modes in nonlinear Hamiltonian system are discussed in [3], namely, force interactions and parametric interactions. We can illustrate the difference between these types of modal interactions using a simple example.

Let us consider two different linear oscillators whose coupling is described by only one anharmonic term, $U=-\gamma \mu_{1}^{2} \mu_{2}$, in the potential energy. Dynamical equations for this system can be written as follows:

$$
\begin{aligned}
& \ddot{\mu}_{1}+\omega_{1}^{2} \mu_{1}=2 \gamma \mu_{1} \mu_{2}, \\
& \ddot{\mu}_{2}+\omega_{2}^{2} \mu_{2}=\gamma \mu_{1}^{2} .
\end{aligned}
$$

Here $\gamma$ is an arbitrary constant characterizing the strength of the interaction of the oscillators. We can suppose that Eqs.(9, 10) describe the dynamics of two modes $\mu_{1}(t)$ and $\mu_{2}(t)$ in a certain mechanical system.

An essential disparity between modes $\mu_{1}(t)$ and $\mu_{2}(t)$ can be seen from the above equations. Indeed, if we excite the mode $\mu_{1}(t)$ at the initial instant $\left(\mu_{1}\left(t_{0}\right) \neq 0\right)$, the mode $\mu_{2}(t)$ cannot be equal to zero (even if it was zero at $t=t_{0}$ !) because a nonzero force $-\frac{\partial U}{\partial \mu_{2}}=\gamma \mu_{1}^{2}$ appears in the right-hand side of Eq.(10) since $\mu_{1}(t) \not \equiv 0$. In other words, the dynamical regime $\mu_{1}(t) \not \equiv 0, \mu_{2}(t) \equiv 0$ cannot exist because of the contradiction with Eq.(10). Unlike 
this, the dynamical regime $\mu_{2}(t) \not \equiv 0, \mu_{1}(t) \equiv 0$ can exist because such a condition does not contradict equations (9) and (10). We can say that now there is no force in the right-hand side of Eq.(10) because $\frac{\partial U}{\partial \mu_{2}} \equiv 0$ as a consequence of the identity $\mu_{1}(t) \equiv 0$.

In the last case, the dynamical regime of the system $(9,10)$ represents a harmonic oscillation only of the second variable:

$$
\mu_{2}(t)=A \cos \left(\omega_{2} t+\delta\right)
$$

where $A$ and $\delta$ are two arbitrary constants.

Thus, there is force action from the mode $\mu_{1}(t)$ on the mode $\mu_{2}(t)$, but not vice versa. We proved in [3] that such a situation can be realized only in the case where the symmetry group of the mode $\mu_{1}(t)$ is less than or equal to that of the mode $\mu_{2}(t)$.

Nevertheless, the mode $\mu_{2}(t)$ can excite the mode $\mu_{1}(t)$ under certain circumstances. Indeed, substituting the solution (11) of Eq.(10) into Eq.(9), we obtain

$$
\ddot{\mu}_{1}(t)+\left[\omega_{1}^{2}-2 A \gamma \cos \left(\omega_{2} t+\delta\right)\right] \mu_{1}=0 .
$$

By means of simple algebraic transformations this equation can be converted to the Mathieu equation in its standard form:

$$
z^{\prime \prime}+[a-2 q \cos (2 \tau)] z=0
$$

where $z=z(\tau)$. But in the $(a-q)$ plane of the Mathieu equation (13), there exist domains of stable and unstable movement. If pertinent parameters $\left(\omega_{1}, \omega_{2}, \gamma\right)$ of our dynamical system (9.10) have values such that corresponding parameters $a$ and $q$ of Eq.(13) get into an unstable domain, then the nonzero function $z(\tau)$ and, therefore, the mode $\mu_{1}(t)$ appears. In other words, the initial dynamical regime $\mu_{1}(t) \equiv 0, \mu_{2}(t) \not \equiv 0$ loses its stability, and a new dynamical regime $\mu_{1}(t) \not \equiv 0, \mu_{2}(t) \not \equiv 0$ arises spontaneously for definite values of the parameters of Eqs.(9,10). Since this phenomenon is similar to the well-known parametric resonance, we can speak, in such a case, about parametric action from the mode $\mu_{2}(t)$ on the mode $\mu_{1}(t)$.

The characteristic property of the parametric interaction is that the appropriate force $\left(-\frac{\partial U}{\partial \mu_{1}}=2 \gamma \mu_{1} \mu_{2}\right.$, in our case) vanishes when the mode $\left(\mu_{1}\right.$, in our case), on which this force acts, becomes zero. The following important result was proven in [3]: the mode of lower symmetry acts on the mode of higher symmetry by force interaction, while the mode of higher symmetry can act on the mode of lower symmetry only parametrically. Consequently, if the parametric excitation of a certain mode does take place, this phenomenon must by necessity be accompanied by spontaneous breaking of symmetry of the mechanical system vibrational state.

Thus, the initially excited dynamical regime can lose its stability because of parametric interactions with some zero modes and, as a result, can transform spontaneously into another dynamical regime, described by a greater number of dynamical variables, with appropriate lowering of symmetry. Obviously, we may treat such phenomenon as a dynamical analog of a phase transition.

The above mechanism of loss of stability of bushes of vibrational modes in octahedral structures with Lennard-Jones interactions will be discussed in Sec.5.

\footnotetext{
${ }^{3}$ Remember, that speaking about a mode we must take into account that our time-dependent coefficient is multiplied by the appropriate basis vector of a certain irrep of the group $G$, and namely this vector determines the symmetry group of the considered mode.
} 


\section{Bushes of vibrational modes for the octahedral struc- ture}

In this section, we consider bushes of vibrational modes for the octahedral structure without a particle in the center of the octahedron depicted in Fig. 1. This mechanical structure, in its equilibrium state, is described by the point symmetry group $O_{h}$, whose brief description and explicit form of the appropriate irreducible representations can be found in Appendix 1. The group $O_{h}$ has ten irreps $\Gamma_{j}(j=1, \ldots, 10)$ : four one-dimensional $\left(\Gamma_{1}, \Gamma_{2}, \Gamma_{3}, \Gamma_{4}\right)$, two two-dimensional $\left(\Gamma_{5}, \Gamma_{6}\right)$ and four three-dimensional. Note that basis vectors (6), as well as invariant vectors (7), depend on the explicit form of the matrix irreps $\Gamma_{j}$ of the group $G=O_{h}$, , but the contributions $\boldsymbol{\Delta}_{j}(8)$ to the vector $\mathbf{X}(t)$ from individual $\Gamma_{j}$ do not depend on the concrete form of these irreps.

\subsection{Basis vectors of the irreps of the group $O_{h}$}

Not all irreps of the group $G$ are contained in the decomposition of the full mechanical representation $\Gamma_{\text {mech }}$ of our system into its irreducible parts. It can be shown that the irreps $\Gamma_{2}, \Gamma_{3}, \Gamma_{4}$ and $\Gamma_{6}$ are not contained in $\Gamma_{\text {mech }}$, while $\Gamma_{10}$ enters into this reducible representation twice. One of two copies of $\Gamma_{10}$ in the decomposition of $\Gamma_{\text {mech }}$ describes the translational movement of the mechanical structure as a rigid body. Considering only vibrational regimes, we will exclude this copy of $\Gamma_{10}$ from consideration. Because of the same reason we also exclude the irrep $\Gamma_{9}$ which describes rotation of the considered octahedral structure. l Thus, there are only $3 \times 6-6=12$ pure vibrational degrees of freedom and, as a consequence, we can restrict ourselves by consideration of the 12-dimensional vibrational representation $\Gamma_{\text {vibr }}$ instead of the 18-dimensional mechanical representation $\Gamma_{\text {mech }}$.

Since decomposition of $\Gamma_{\text {vibr }}$ contains only one copy of each irrep $\Gamma_{j}$ for $j=1,5,7,8,10$, all symmetry adapted coordinates, generated by the basis vectors of these irreps, turn out to be the normal coordinates, and we may not distinguish these two notations in the further discussion.

We give the basis vectors of the irreps $\Gamma_{j}$, which form a basis of the vibrational representation $\Gamma_{\text {vibr }}$, in Table 3. These basis vectors were found by the well-known method of projection operators with the aid of the appropriate computer program. Each of these 18dimensional vectors determines the specific set of displacements $\left(\mathbf{r}_{1}\left|\mathbf{r}_{2}\right| \mathbf{r}_{3}\left|\mathbf{r}_{4}\right| \mathbf{r}_{5} \mid \mathbf{r}_{6}\right)$ of the six particles of our octahedral structure (the numbers of particles are given in Fig. 1). Here $\mathbf{r}_{j}=\left(x_{j}, y_{j}, z_{j}\right)$ is the three-dimensional displacement vector of the $j$-th particle from its equilibrium position determined by its projections along three Cartesian axes $X, Y, Z$. The complete set of basis vectors $\varphi_{i}^{(j)}$ from Table 3 can be used as a basis for the decomposition of an arbitrary vibrational regime $\mathbf{X}(t)$ (see Eq.(2)).

\subsection{Bushes of vibrational modes}

As was already discussed in previous sections, a certain symmetry group $G_{D} \subseteq G$ corresponds to a given bush. Therefore, to find all bushes, we must know all subgroups $G_{D}^{(k)}$ of the parent group $G=O_{h}$ of our mechanical system in its equilibrium state. Moreover, we must take into

\footnotetext{
${ }^{4}$ Namely because of this reason we give the explicit form of the irreps of the group $O_{h}$ in Appendix 1 (remember that a given irrep is determined up to arbitrary unitary transformation).

${ }^{5}$ There exist also rotational-vibrational bushes for which modes of $\Gamma_{9}$ turn to be root modes, but in the present paper, we will study only pure vibrational bushes.
} 
Table 3: Basis vectors of the irreps of the group $O_{h}$ for the octahedral structure depicted in Fig.1

\begin{tabular}{|c|c|c|}
\hline & Basis vectors & Symmetry groups \\
\hline $\boldsymbol{\varphi}_{1}^{(1)}$ & $\frac{1}{\sqrt{6}}(0,0,1|-1,0,0| 0,1,0|1,0,0| 0,-1,0 \mid 0,0,-1)$ & $O_{h}$ \\
\hline $\boldsymbol{\varphi}_{1}^{(5)}$ & $\frac{1}{\sqrt{12}}(0,0,-2|-1,0,0| 0,1,0|1,0,0| 0,-1,0 \mid 0,0,2)$ & $D_{4 h}$ \\
\hline $\boldsymbol{\varphi}_{2}^{(5)}$ & $\frac{1}{\sqrt{12}}(0,0,0|\sqrt{3}, 0,0| 0, \sqrt{3}, 0|-\sqrt{3}, 0,0| 0,-\sqrt{3}, 0 \mid 0,0,0)$ & $D_{2 h}$ \\
\hline $\boldsymbol{\varphi}_{1}^{(7)}$ & $\frac{1}{2}(0,1,0|0,0,0| 0,0,1|0,0,0| 0,0,-1 \mid 0,-1,0)$ & $D_{2 h}^{\prime}$ \\
\hline $\boldsymbol{\varphi}_{2}^{(7)}$ & $\frac{1}{2}(1,0,0|0,0,-1| 0,0,0|0,0,1| 0,0,0 \mid-1,0,0)$ & $D_{2 h}^{\prime}$ \\
\hline $\boldsymbol{\varphi}_{3}^{(7)}$ & $\frac{1}{2}(0,0,0|0,-1,0| 1,0,0|0,1,0|-1,0,0 \mid 0,0,0)$ & $D_{2 h}^{\prime}$ \\
\hline $\boldsymbol{\varphi}_{1}^{(8)}$ & $\frac{1}{2}(1,0,0|0,0,0|-1,0,0|0,0,0|-1,0,0 \mid 1,0,0)$ & $D_{2 d}^{\prime}$ \\
\hline $\boldsymbol{\varphi}_{2}^{(8)}$ & $\frac{1}{2}(0,-1,0|0,1,0| 0,0,0|0,1,0| 0,0,0 \mid 0,-1,0)$ & $D_{2 d}^{\prime}$ \\
\hline $\boldsymbol{\varphi}_{3}^{(8)}$ & $\frac{1}{2}(0,0,0|0,0,-1| 0,0,1|0,0,-1| 0,0,1 \mid 0,0,0)$ & $D_{2 d}^{\prime}$ \\
\hline $\boldsymbol{\varphi}_{1}^{(10)}$ & $\frac{1}{\sqrt{12}}(1,0,0|-2,0,0| 1,0,0|-2,0,0| 1,0,0 \mid 1,0,0)$ & $C_{4 v}$ \\
\hline $\boldsymbol{\varphi}_{2}^{(10)}$ & $\frac{1}{\sqrt{12}}(0,1,0|0,1,0| 0,-2,0|0,1,0| 0,-2,0 \mid 0,1,0)$ & $C_{4 v}$ \\
\hline $\boldsymbol{\varphi}_{3}^{(10)}$ & $\frac{1}{\sqrt{12}}(0,0,-2|0,0,1| 0,0,1|0,0,1| 0,0,1 \mid 0,0,-2)$ & $C_{4 v}$ \\
\hline
\end{tabular}

account not only different subgroups, but also their different embeddings (orientations) in the parent group $O_{h}$. For example, there are three ways of embedding of the group $G_{D}=C_{2 v}$ (we denote them by primes on the appropriate Schoenflies symbols: $C_{2 v}^{\prime}, C_{2 v}^{\prime \prime}$ ) - they differ from each other by their generators. We give the generators of all subgroups of the group $O_{h}$, corresponding to different nonlinear vibrational regimes, in the second column of Tables 1.2. For the above case we see the following generators for three variants of the subgroup $C_{2 v}$ :

$$
C_{2 v}: h_{4}, h_{26} ; \quad C_{2 v}^{\prime}: h_{13}, h_{28} ; \quad C_{2 v}^{\prime \prime}: h_{4}, h_{37}
$$

Using the list of symmetry elements from [9], one can find that in $C_{2 v}$ the two-fold axis $h_{4}$ is the coordinate axis $C_{2}^{z}$ and the mirror plane $h_{26}$ is the coordinate plane $\sigma_{x} ;$ in $C_{2 v}^{\prime}, h_{13}$ is the diagonal two-fold axis $C_{2}^{\bar{x} y}$, while the mirror plane $h_{28}$ is the coordinate plane $\sigma_{z}$; in $C_{2 v}^{\prime \prime}$, $h_{4}$ is the coordinate axis $C_{2}^{z}$ and the mirror plane $h_{37}$ coincides with the diagonal plane $\sigma_{\bar{x} y}$.

All subgroups $G_{D}^{(k)}$ of the group $G=O_{h}$ with their different embeddings and the sets of appropriate invariant vectors for each irrep of this group can be found in 10.6

Let us remember, that for obtaining a given bush of vibrational modes with symmetry group $G_{D} \subseteq G$, we must solve the linear algebraic equations (5) for each of five irreps $\Gamma_{j}(j=1,5,7,8,10)$ of the group $G=O_{h}$. In such a way, we obtain the invariant vector $\boldsymbol{\mu}_{j}$ individually for every irrep $\Gamma_{j}$. It is very essential that for many irreps these invariant vectors turn out to be zero vectors. For example, for $G_{D}=D_{4 h}$ there are only two irreps to which nonzero invariant vectors correspond: the one-dimensional irrep $\Gamma_{1}$ and the two-dimensional irrep $\Gamma_{5}$. Moreover, the invariant vector for $\Gamma_{5}$ has a special form: $\boldsymbol{\mu}_{5}=(a, 0)$, where $a$ is an arbitrary constant appearing in the solution of Eq.(5). Therefore, we obtain the following expression for the bush $B 2\left[D_{4 h}\right]$, if replacing the arbitrary constants by time-dependent

\footnotetext{
${ }^{6}$ The invariant vectors of irreps of group $O_{h}$ were obtained in the paper [10] in connection with discussion of some problems of the theory of color symmetry.

${ }^{7} \mathrm{~A}$ reader can see this result in Table 1 .
} 
functions $\mu_{i}^{(j)}(t)$ :

$$
\mathbf{X}(t)=\mu_{1}^{(1)}(t) \boldsymbol{\varphi}_{1}^{(1)}+\mu_{1}^{(5)}(t) \boldsymbol{\varphi}_{1}^{(5)} .
$$

(Note that the coefficient before the second basis vector $\varphi_{2}^{(5)}$ of the irrep $\Gamma_{5}$ is equal to zero!). Analogously, for bush $B 3\left[D_{2 d}^{\prime}\right]$, we find that only three irreps $\Gamma_{1}, \Gamma_{5}$ and $\Gamma_{8}$ contribute to the corresponding dynamical regime:

$$
\mathbf{X}(t)=\mu_{1}^{(1)}(t) \boldsymbol{\varphi}_{1}^{(1)}+\mu_{1}^{(5)}(t) \boldsymbol{\varphi}_{1}^{(5)}+\mu_{3}^{(8)}(t) \boldsymbol{\varphi}_{3}^{(8)} .
$$

Again, we see that a very specific invariant vector $\boldsymbol{\mu}_{8}=(0,0, a)$ corresponds to the threedimensional irrep $\Gamma_{8}$.

Comparing Eqs.(15) and (16), one finds the identical symbols $\mu_{1}^{(1)}(t)$ and $\mu_{1}^{(5)}(t)$ in two different bushes $B 2\left[D_{4 h}\right]$ and $B 3\left[D_{2 d}\right]$. There is no connection between these functions, because we exploit here, as well as in Tables 1,2, the following convention: one and the same notation $\mu_{i}^{(j)}(t)$ corresponds to different functions when it is used in the description of different bushes.

Let us consider Tables 1 and 2 where all bushes of vibrational modes for the octahedral mechanical structure are listed. The root modes of bushes are underlined (remember, that root mode possesses the minimal symmetry among all other modes contained in the given bush)

Similar to phase transition theory, we can speak about dynamical domains for a given bush. Indeed, as it can be seen from Tables 1.2, there is only one bush with the symmetry group $G_{D}=D_{4 h}$, namely, the bush $B 2\left[D_{4 h}\right]$. The 4 -fold axis of this group, associated with the generator $h_{14}$, is directed along the $Z$ coordinate axis, i.e., $G_{D}=D_{4 h}^{z}$ (see geometrical sense of the generators from the third column of Table 1 in the handbook [6]). Obviously, there must exist also bushes with the same symmetry group $D_{4 h}$, but with 4 -fold axis oriented along $X$ and $Y$ coordinate axis, i.e. $B\left[D_{4 h}^{x}\right]$ and $B\left[D_{4 h}^{y}\right]$, because of the equal status of the $X, Y, Z$ axes in the parent group $G=O_{h}$. The collection of these three equivalen 10 bushes is similar to the set of different domains in the phase transition theoryet. In Tables 1,2 we include only one bush from the complete collection of the equivalent bushes.

As it can be seen from Tables 1,2, the root modes corresponding to some bushes represent superpositions of several basis vectors of a given multidimensional irrep. For example, we find in the fifth column of this table that the root modes for bushes $B 7\left[D_{3}\right], B 8\left[C_{3 v}\right]$ and $B 9\left[D_{3 d}\right]$ are simply sums of all three basis vectors of the irreps $\Gamma_{8}, \Gamma_{10}$ and $\Gamma_{7}$, respectively. In terms of invariant vectors, this means that in solving Eq.(5) we obtain for the above irreps the solutions of the same form: $\boldsymbol{\mu}_{8}=\boldsymbol{\mu}_{10}=\boldsymbol{\mu}_{7}=(a, a, a)$, where $a$ is an arbitrary constant (this constant is different for each of the irreps $\Gamma_{8}, \Gamma_{10}$ and $\Gamma_{7}$ !). In writing the bushes $B 7\left[D_{3}\right], B 8\left[C_{3 v}\right]$ and $B 9\left[D_{3 d}\right]$ we must replace these constants by the appropriate functions of time $\mu_{1}^{(8)}(t), \mu_{1}^{(10)}(t)$ and $\mu_{1}^{(7)}(t)$, respectively.

Let us now consider the more complicated cases where the choice of root mode for a given bush is not uniquely defined. For example, there are two different variants for choosing the root mode for bush $B 6\left[C_{2 v}^{\prime}\right]$ : either the sum $\left(\varphi_{1}^{(8)}+\varphi_{2}^{(8)}\right)$ of two basis vectors of threedimensional irrep $\Gamma_{8}$, or the difference $\left(\varphi_{1}^{(10)}-\varphi_{2}^{(10)}\right)$ of two basis vectors of another three-

\footnotetext{
${ }^{8} \mathrm{~A}$ more exact discussion of this topic see below.

${ }^{9}$ Two bushes are equivalent to each other, if there exist a symmetry element $g$ of the parent group $G$ which transforms one bush to another.

${ }^{10}$ The total number of such domains is equal to the index of the subgroup $G_{D}$ in the group $G$, i.e., to the ratio of the orders of these two groups: $\|G\| /\left\|G_{D}\right\|$.
} 
dimensional irrep $\Gamma_{10}$ can represent the root mode.11 Indeed, the excitation of each of these modes separately or simultaneously leads to the excitation of the same bush $B 6\left[C_{2 v}^{\prime}\right]$.

Such a possibility of different choices of the root mode is brought about by the fact that both combinations of the basis vectors $\left(\varphi_{1}^{(8)}+\varphi_{2}^{(8)}\right)$ and $\left(\varphi_{1}^{(10)}-\varphi_{2}^{(10)}\right)$ possess the same symmetry group $G_{D}=C_{2 v}^{\prime}$, while the basis vectors of other irreps contributing to bush $B 6\left[C_{2 v}^{\prime}\right]$ have higher symmetry, namely, $\varphi_{1}^{(1)}-O_{h}, \boldsymbol{\varphi}_{1}^{(5)}-D_{4 h}, \boldsymbol{\varphi}_{3}^{(7)}-D_{2 h}^{\prime}$ (these three point groups are supergroups with respect to the symmetry group $C_{2 v}^{\prime}$ of the root modes). From this example we see that all modes of a given bush do have symmetry higher than or equal to that of the root mode (see Sec. 2.1).

Above, we consider the cases where bushes may be excited by the initial excitation of a single mode (even if there are different variants of the choice of this single root mode). But there exists bushes of another type, namely, the bushes whose excitation is possible only if two different modes, i.e. modes belonging to the different irreps, are excited simultaneously. For the considered mechanical system there are four bushes of such a type in Table 2:

$$
B 11\left[D_{2}^{\prime}\right]-\Gamma_{7}, \Gamma_{8} ; B 12\left[C_{2 v}\right]-\Gamma_{8}, \Gamma_{10} ; B 13\left[C_{2 v}^{\prime \prime}\right]-\Gamma_{7}, \Gamma_{10} ; B 17\left[C_{3}\right]-\Gamma_{8}, \Gamma_{10} \text {. }
$$

Here we also point out the pairs of the irreps whose modes must be excited for the excitation of the corresponding bushes.

Let us consider the bush $B 13\left[C_{2 v}^{\prime \prime}\right]$ in more detail. We can write down the symmetry groups of all four modes contributing to it (these groups can be found in Table 3):

$$
\boldsymbol{\varphi}_{1}^{(1)}-O_{h}, \boldsymbol{\varphi}_{1}^{(5)}-D_{4 h}, \boldsymbol{\varphi}_{3}^{(7)}-D_{2 h}^{\prime}, \boldsymbol{\varphi}_{3}^{(10)}-C_{4 v}
$$

Note that the symmetry group $C_{2 v}^{\prime \prime}$ of the whole bush $B 13\left[C_{2 v}^{\prime \prime}\right]$ is not found among the symmetry groups (18) of its individual modes. This fact can be understood as follows. Since our bush is the superposition of four modes $\varphi_{1}^{(1)}, \varphi_{1}^{(5)}, \varphi_{3}^{(7)}$ and $\varphi_{3}^{(10)}$, it is clear that its symmetry group must be the intersection of the four symmetry groups corresponding to these modes:

$$
O_{h}\left(h_{2}, h_{3}, h_{5}, h_{13}, h_{25}\right), D_{4 h}\left(h_{2}, h_{14}, h_{25}\right), D_{2 h}^{\prime}\left(h_{4}, h_{13}, h_{25}\right), C_{4 v}\left(h_{14}, h_{37}\right) \text {. }
$$

Here we write the generators of each group in the parentheses next to its Schoenflies symbol (these generators can be found in the third column of Table 1 near the appropriate symbol of the symmetry group) 12 .

The two first groups from (19) - $O_{h}$ and $D_{4 h}$ - are subgroups with respect to of the both last groups, $D_{2 h}^{\prime}$ and $C_{4 v}$ : all elements of $D_{2 h}^{\prime}$ and $C_{4 v}$ are contained in $D_{4 h}$ and therefore also in $O_{h}$. Indeed, the group $D_{4 h}\left(h_{2}, h_{14}, h_{25}\right)$ contains four two-fold horizontal axes (rotations $h_{2}, h_{3}, h_{13}, h_{17}$ ) and four vertical mirror planes (reflections $h_{26}, h_{27}, h_{37}, h_{41}$ ) intersecting along vertical four-fold axis (rotations $h_{4}, h_{14}, h_{15}$ ) coinciding with the $Z$ coordinate axis, and it is obvious that the generators of both groups $D_{2 h}^{\prime}$ and $C_{4 v}$ are identical with some elements of the group $D_{4 h} 13$.

Let us now compare the elements of the groups $D_{2 h}^{\prime}\left(h_{4}, h_{13}, h_{25}\right)$ and $C_{4 v}\left(h_{14}, h_{37}\right)$. The second generator of $C_{4 v}$ is contained in $D_{2 h}^{\prime}$ since $h_{37}=h_{13} \cdot h_{25}$, and the first generator of $D_{2 h}^{\prime}$ is contained in $C_{4 v}$ since $h_{14}^{2}=h_{4}$ (see footnote 13). Then we can write the extended sets

\footnotetext{
${ }^{11}$ The invariant vectors $(a, a, 0)$ and $(a,-a, 0)$ correspond to these two cases.

${ }^{12}$ Only for group $O_{h}$, we use the extended set of generators.

${ }^{13}$ Using the multiplication table from [9] for the element of the group $O_{h}$, we can obtain the generators of $D_{2 h}^{\prime}$ and $C_{4 v}$ from the generators of $D_{4 h}$ as follows: $h_{4}=h_{14}^{2}, h_{13}=h_{2} h_{14}, h_{37}=h_{13} h_{25}$.
} 
of the generators of the groups $C_{4 v}$ and $D_{2 d}^{\prime}$ as follows: $C_{4 v}\left(h_{14}, \underline{h_{4}, h_{37}}\right), D_{2 d}^{\prime}\left(h_{13}, h_{25}, \underline{h_{4}, h_{37}}\right)$. The intersection of these two groups is a group with their common elements, i.e., the group with the generators $h_{4}$ and $h_{37}$. In our notation, this is the group $C_{2 v}^{\prime \prime}\left(h_{4}, h_{37}\right)$ associated with the whole bush $B 13\left[C_{2 v}^{\prime \prime}\right]$ in Table 2 .

Other three bushes from the list (17) can be considered similarly to the above discussed bush $B 13\left[C_{2 v}^{\prime \prime}\right]$.

Unlike the above cases, the modes of only one irrep are root modes for bushes

$$
B 10\left[D_{2 h}\right], B 14\left[C_{2}^{\prime}\right], B 16\left[C_{s}^{\prime}\right], B 18\left[C_{i}\right]
$$

but these modes are determined by several independent coefficients $\mu_{i}^{(j)}(t)$. For example, the root mode $\mu_{1}^{(5)}(t) \boldsymbol{\varphi}_{1}^{(5)}+\mu_{2}^{(5)}(t) \boldsymbol{\varphi}_{2}^{(5)}$ corresponds to the bush $B 10\left[D_{2 h}\right]$ (This mode is generated by the invariant vector of the form $(a, b)$, associated with the two-dimensional irrep $\left.\Gamma_{5}\right)$. Analogously, the root mode $\mu_{1}^{(10)}(t)\left(\varphi_{1}^{(10)}+\varphi_{2}^{(10)}\right)+\mu_{3}^{(10)}(t) \varphi_{3}^{(10)}$ corresponds to the bush $B 16\left[C_{s}^{\prime}\right]$ (this mode is induced by the invariant vector of the form $(a, a, b)$ of the three-dimensional irrep $\left.\Gamma_{10}\right)$.

\section{Exact dynamical equations for bushes of vibrational modes}

We consider a mechanical system of six mass points (particles) whose interactions are described by a pair isotropic potential $u(r)$ where $r$ is the distance between two particles. We suppose that in the equilibrium state these particles form a regular octahedron with edge $a_{0}$ which is depicted in Fig. 1. Let us introduce a Cartesian coordinate system. Four particles of the above octahedron lie in the $X Y$ plane and form a square with edge $a_{0}$. Two other particles lie on the $Z$ axis and we will speak about the "top particle" and the "bottom particle" with respect to the direction of the $Z$ axis. Obviously, the distance between each of these two particles and any of the four particles in the $X Y$ plane is equal to $a_{0}$.

In the equilibrium state, our mechanical system possesses the point symmetry group $O_{h}$. All possible bushes of vibrational modes were considered for this system in the previous section, and we remember that a certain subgroup $G_{D}$ of the group $G=O_{h}$ corresponds to each of these bushes.

In this section, we consider $u(r)$ as an arbitrary pair isotropic potential, but for studying the bush stability in Sec.5 we suppose that $u(r)$ is the well-known Lennard-Jones potential:

$$
u(r)=\frac{A}{r^{12}}-\frac{B}{r^{6}}
$$

Here $A$ and $B$ are certain constants characterizing the value of the repulsive and attractive potential parts, respectively.

The potential energy of our system in its vibrational state can be written in the form

$$
V(\mathbf{X})=\sum_{\substack{i, j \\(i<j)}} u\left(r_{i j}\right),
$$

where $r_{i j}$ is a distance between the $i$-th and $j$-th particles. The $N$-dimensional vector $\mathbf{X}=\left(x_{1}(t), x_{2}(t), \ldots, x_{N}(t)\right)$ in Eq. (22), already introduced in Sec.2, determines the dis- 
placements of all particles at an arbitrary chosen instant $t$. Note that in our present case $N=18.14$

Since all particles possess identical masses, which we suppose to be equal to unity, the dynamical equations of Newton type can be written as follows:

$$
\ddot{x}_{i}=-\frac{\partial V}{\partial x_{i}}, \quad i=1, \ldots, N \quad(N=18) .
$$

It is easy to find such a scaling transformation that both constants $A$ and $B$ in the potential energy $V(\mathbf{X})$ (see Eqs.(21, 22) will be equal to unity $(A=1, B=1)$ :

$$
\begin{aligned}
& x_{i}=\left(\frac{A}{B}\right)^{\frac{1}{6}} \tilde{x}_{i}, \\
& t=\frac{m^{\frac{1}{2}} A^{\frac{2}{3}}}{B^{\frac{7}{6}}} \tilde{t} .
\end{aligned}
$$

Because of this transformation, there are no arbitrary constants in Eq.(23) and, therefore, the results obtained in the present paper are universal with respect to all Lennard-Jones potentials. Supposing that the scaling transformation (24) is already done, we will not change the notation of the time variable $t$ and space variables $x_{i}$ in the further discussion.

\subsection{Lagrange equations for bush modes}

For obtaining dynamical equations of bushes of modes, we must transfer from the original (old) variables $x_{i}(t)(i=1,2, \ldots, N)$ to new variables $\mu_{j}(t)(j=1,2, \ldots, N)$, which describe the amplitudes of modes in the decomposition (2)

$$
\mathbf{X}(t)=\sum_{j=1}^{N} \mu_{j}(t) \boldsymbol{\varphi}_{j}
$$

In contrast to Eq.(2) we use here a single index $j$ for numbering the basis vectors of all irreps of the group $G$.

When a certain bush with a symmetry group $G_{D} \subset G$ is considered, some amplitudes $\mu_{j}(t)$ turn out to be equal to zero $\left(\mu_{j}(t) \equiv 0\right)$ and we can suppose without loss of generality that index $j$ from Eq.(25) runs over only the first $n$ values $(j=1,2, \ldots, n)$. Here $n<N$ for all bushes of modes except for the bush with trivial symmetry group $G_{D}=1$. Thus, a given bush represents a dynamical system whose dimension is less than that of the original mechanical system $(n<N)$ and its time evolution can be described with the aid of $n$ generalized variables $\mu_{j}(t)(j=1, \ldots, n)$. The Lagrange method seems to be the most natural and convenient for obtaining the dynamical equations for bushes of modes. Let us consider this idea in more detail.

For a given $n$-dimensional bush we can rewrite Eq.(25) in the form

$$
\mathbf{X}(t)=\sum_{j=1}^{n} \mu_{j}(t) \boldsymbol{\varphi}_{j}
$$

Since all basis vectors $\boldsymbol{\varphi}_{j}$ are known, Eq. (26) provides explicit expression of each of the $N$ old variables $x_{i}(t)(i=1, \ldots, N)$ as a function (linear superposition!) of $n$ new variables

\footnotetext{
${ }^{14}$ In this section, we denote by $N$ the total number of degrees of freedom of the considered mechanical system (in other sections, $N$ is the number of particles).
} 
$\mu_{j}(t)(j=1, \ldots, n)$. Therefore, the potential energy $\widetilde{V}\left(\mu_{1}, \ldots, \mu_{n}\right)$ in the new variables can be obtained from the potential energy $V\left(x_{1}, \ldots, x_{N}\right)$ in the old variables by substitution $x_{i}(t)(i=1, \ldots, N)$ from Eq.(26) to the function $V\left(x_{1}, \ldots, x_{N}\right)$. Similarly, the kinetic energy $T\left(\dot{x}_{1}, \ldots, \dot{x}_{N}\right)=\frac{1}{2} \sum_{i=1}^{N} \dot{x}_{i}^{2}$ can be transformed with the aid of Eq.26) to the following quadratic form in terms of velocities $\dot{\mu}_{j}(t)$ of the new variables:

$$
\widetilde{T}\left(\dot{\mu}_{1}, \ldots, \dot{\mu}_{n}\right)=\frac{1}{2} \sum_{j=1}^{n} \sum_{k=1}^{n} \dot{\mu}_{j} \dot{\mu}_{k}\left\langle\boldsymbol{\varphi}_{j} \mid \boldsymbol{\varphi}_{k}\right\rangle .
$$

Here $\left\langle\boldsymbol{\varphi}_{j} \mid \boldsymbol{\varphi}_{k}\right\rangle$ is the scalar product of two real $N$-dimensional basis vectors $\boldsymbol{\varphi}_{j}$ and $\boldsymbol{\varphi}_{k}$. We can suppose that the basis vectors $\boldsymbol{\varphi}_{j}(j=1, \ldots, N)$ are orthonormal:

$$
\left\langle\boldsymbol{\varphi}_{j} \mid \boldsymbol{\varphi}_{k}\right\rangle=\delta_{j k}
$$

(Note that basis vectors from Table 3 do satisfy this condition).

Taking into account Eq.(28), we can rewrite Eq.(27) as follows:

$$
\widetilde{T}\left(\dot{\mu}_{1}, \ldots, \dot{\mu}_{n}\right)=\frac{1}{2} \sum_{j=1}^{n} \dot{\mu}_{j}^{2}
$$

Then with the aid of the Lagrange function $\widetilde{L}=\widetilde{T}-\widetilde{V}$, the exact dynamical equations for a given $n$-dimensional bush with the symmetry group $G_{D} \subset G$ can be obtained:

$$
\frac{d}{d t}\left(\frac{\partial \widetilde{L}}{\partial \dot{\mu}_{j}}\right)-\frac{\partial \widetilde{L}}{\partial \mu_{j}}=0 \quad(j=1, \ldots, n) .
$$

Let us emphasize that these dynamical equations of the bush of modes are exact. Actually, this fact is brought about by the symmetry-related restriction

$$
\widehat{G}_{D} \mathbf{X}(t)=\mathbf{X}(t)
$$

which reduces the number of degrees of freedom from $N$ to $n$. Indeed, namely the condition (31) leads to the vanishing of some terms in Eq.(25) and reduces it to Eq.(26).

\subsection{Examples of dynamical equations for bushes of vibrational modes}

The exact equations (30) for bushes of vibrational modes for the octahedral structure with the Lennard-Jones potential are rather complicated. Because of this reason, we wrote a special MAPLE-program for obtaining these equations and for studying the stability of bushes of modes in modal space (see Sec. 5.3). Let us now write down the very clear and compact forms of the exact dynamical equations for some bushes in terms of natural geometrical variables.

We consider the following three bushes from Table 1: $B 1\left[O_{h}\right], B 2\left[D_{4 h}\right]$ and $B 4\left[C_{4 v}\right]$, whose dimension is equal to 1,2 and 3 , respectively. Note that symmetry groups of the above bushes are connected to each other by the following group-subgroup relation: $C_{4 v} \subset D_{4 h} \subset O_{h}$.

The geometrical forms of our mechanical system in the vibrational state, corresponding to the these bushes, can be revealed from the superpositions of modes (given in the fifth column of Table 1). But it is easier to understand the geometrical sense of the above bushes immediately from the symmetry groups corresponding to them. 
Indeed, the one-dimensional bush $B 1\left[O_{h}\right]$ consists of only one ("breathing") mode: the appropriate nonlinear dynamical regime $\mathbf{X}(t)=\mu_{1}^{(1)}(t) \boldsymbol{\varphi}_{1}^{(1)}$ describes evolution of a regular octahedron whose edge $a=a(t)$ periodically changes in time.

The two-dimensional bush $B 2\left[D_{4 h}\right]$ describes a dynamical regime with two degrees of freedom: $\mathbf{X}(t)=\mu_{1}^{(1)}(t) \boldsymbol{\varphi}_{1}^{(1)}+\mu_{1}^{(5)}(t) \boldsymbol{\varphi}_{1}^{(5)}$. The symmetry group $G_{D}=D_{4 h}$ of this bush contains the 4-fold axis coinciding with the $Z$ coordinate axis and the mirror plane coinciding with the $X Y$ plane. This symmetry group restricts essentially the form of the polyhedron describing our mechanical system in the vibrational state. Indeed, the presence of the 4-fold axis demands that the quadrangle in the $X Y$ plane be a square. Because of the same reason, the four edges connecting the particles in the $X Y$ plane (vertices of the above square) with the top particle lying on the $Z$ axis must be of the same length which we denote by $b(t)$.

Similarly, let the length of the edges connecting the bottom particle on the $Z$ axis with any of the 4 particles in the $X Y$ plane be denoted by $c(t)$. In our present case of the bush $B 2\left[D_{4 h}\right], b(t)=c(t)$ for any time $t$ because of the presence of the horizontal mirror plane in the group $G_{D}=D_{4 h}$. But for the three-dimensional bush $B 4\left[C_{4 v}\right]$, described by $\mathbf{X}(t)=\mu_{1}^{(1)}(t) \boldsymbol{\varphi}_{1}^{(1)}+\mu_{1}^{(5)}(t) \boldsymbol{\varphi}_{1}^{(5)}+\mu_{3}^{(10)}(t) \boldsymbol{\varphi}_{3}^{(10)}$, this mirror plane is absent and, therefore, $b(t) \neq c(t)$.

Let us also introduce two heights, $h_{1}(t)$ and $h_{2}(t)$, corresponding to the perpendiculars dropped, respectively, from the top and bottom vertices of our polyhedron onto the $X Y$ plane. Now we can write the dynamical equations of the above bushes in terms of pure geometrical variables $a(t), b(t), c(t), h_{1}(t)$ and $h_{2}(t)$.

We choose $a(t)$ and $h(t) \equiv h_{1}(t) \equiv h_{2}(t)$ as dynamical variables for describing the twodimensional bush $B 2\left[D_{4 h}\right]$ and $a(t), h_{1}(t)$ and $h_{2}(t)$ as dynamical variables for describing the three-dimensional bush $B 4\left[C_{4 v}\right]$. Using these variables we can write down the potential energy for our bushes of vibrational modes as follows:

$$
\begin{array}{lll}
B 1\left[O_{h}\right] & : & V_{B 1}(a)=12 u(a)+3 u(\sqrt{2} a), \\
B 2\left[D_{4 h}\right]: & V_{B 2}(a, h)=4 u(a)+2 u(\sqrt{2} a)+8 u\left(\sqrt{h^{2}+\frac{a^{2}}{2}}\right)+u(2 h), \\
B 4\left[C_{4 v}\right]: & V_{B 4}\left(a, h_{1}, h_{2}\right)=4 u(a)+2 u(\sqrt{2} a)+4 u(b)+4 u(c)+u\left(h_{1}+h_{2}\right),
\end{array}
$$

where $b=\sqrt{\frac{a^{2}}{2}+\left(\frac{5}{4} h_{1}-\frac{1}{4} h_{2}\right)^{2}}, c=\sqrt{\frac{a^{2}}{2}+\left(\frac{5}{4} h_{2}-\frac{1}{4} h_{1}\right)^{2}}$.

Then with the aid of the Lagrange method, we can obtain the following dynamical equations for the above bushes of vibrational modes:

$$
\begin{aligned}
B 1\left[O_{h}\right]: & \\
\ddot{a}= & -4 u^{\prime}(a)-\sqrt{2} u^{\prime}(\sqrt{2} a) ; \\
B 2\left[D_{4 h}\right]: & \\
\ddot{a}= & -2 u^{\prime}(a)-\sqrt{2} u^{\prime}(\sqrt{2} a)-2 u^{\prime}(b) \frac{a}{b}, \\
\ddot{h}= & -4 u^{\prime}(b) \frac{h}{b}-u^{\prime}(2 h) ; \\
B 4\left[C_{4 v}\right]: & \\
\ddot{a}= & -2 u^{\prime}(a)-\sqrt{2} u^{\prime}(\sqrt{2} a)-u^{\prime}(b) \frac{a}{b}-u^{\prime}(c) \frac{a}{c}, \\
\ddot{h_{1}}= & -u^{\prime}(b) \frac{5 h_{1}-h_{2}}{b}-u^{\prime}\left(h_{1}+h_{2}\right), \\
\ddot{h_{2}}= & -u^{\prime}(c) \frac{5 h_{2}-h_{1}}{c}-u^{\prime}\left(h_{1}+h_{2}\right) .
\end{aligned}
$$

Thus, we obtain the dynamical equations of our bushes of vibrational modes in terms of variables with explicit geometrical sense. Each bush describes a certain nonlinear dynamical regime corresponding to such a vibrational state of the considered mechanical system, that 
at any fixed time the configuration of this system is represented by a definite polyhedron with symmetry group $G_{D}$ of the given bush.

We can write dynamical equations for the above bushes in terms of vibrational modes as well. In spite of the more complicated form, these equations turn out to be more useful for the bush theory, since they allow us to decompose the appropriate nonlinear dynamical regimes into modes of different importance for the case of small oscillations - root modes and secondary modes of different orders [3]. As an example, we write below the dynamical equations for the bush $B 4\left[C_{4 v}\right]$ in terms of its three modes, $\mu_{3}^{(10)}(t) \equiv \gamma(t)$ (root mode), $\mu_{1}^{(1)}(t) \equiv \mu(t), \mu_{1}^{(5)}(t) \equiv \nu(t)$ (secondary modes):

$$
\begin{aligned}
\ddot{\mu}= & -\frac{1}{6}\left(4 \sqrt{2} u^{\prime}(a)+4 u^{\prime}(\sqrt{2} a)+2 u^{\prime}\left(h_{1}+h_{2}\right)+\frac{u^{\prime}(b)}{b}\left(2 \sqrt{2} a+5 h_{1}-h_{2}\right)+\right. \\
& \left.\frac{u^{\prime}(c)}{c}\left(2 \sqrt{2} a+5 h_{2}-h_{1}\right)\right), \\
\ddot{\nu}= & -\frac{1}{6}\left(2 \sqrt{2} u^{\prime}(a)+2 u^{\prime}(\sqrt{2} a)-2 u^{\prime}\left(h_{1}+h_{2}\right)+\frac{u^{\prime}(b)}{b}\left(\sqrt{2} a-5 h_{1}+h_{2}\right)+\right. \\
& \left.\frac{u^{\prime}(c)}{c}\left(\sqrt{2} a-5 h_{2}+h_{1}\right)\right), \\
\ddot{\gamma}= & \frac{1}{4}\left(u^{\prime}(b) \frac{5 h_{1}-h_{2}}{b}-u^{\prime}(c) \frac{5 h_{2}-h_{1}}{c}\right) .
\end{aligned}
$$

Here

$$
\begin{gathered}
a=\sqrt{2}\left(r_{0}+\mu+\nu\right) ; b=\sqrt{\left(r_{0}+\mu+\nu\right)^{2}+\left(r_{0}+\mu-2 \nu-3 \gamma\right)^{2}} ; \\
c=\sqrt{\left(r_{0}+\mu+\nu\right)^{2}+\left(r_{0}+\mu-2 \nu+3 \gamma\right)^{2}} ; \\
h_{1}=r_{0}+\mu-2 \nu-2 \gamma ; h_{2}=r_{0}+\mu-2 \nu+2 \gamma ; r_{0}=\frac{a_{0}}{\sqrt{2}} .
\end{gathered}
$$

Let us stress that all above equations are valid not only for the Lennard-Jones potential (21), but for any pair and isotropic potential $u(r)$, as well.

Now, we want to demonstrate that Eqs. 33, 34, 35) possess the specific bush structure which is discussed in Sec2. Note that there are the definite relations between the symmetry groups of the modes $\mu(t), \nu(t)$ and $\gamma(t)$ :

$$
G_{D}[\mu]=O_{h} \supset G_{D}[\nu]=D_{4 h} \supset G_{D}[\gamma]=C_{4 v}
$$

(see Tables 3 and 11). Therefore, it follows from the general bush theory that the mode $\gamma(t)$, being of minimal symmetry, must provide some forces in the right-hand side (rhs) of the Eqs.(33) and (34) even if $\mu(t) \equiv 0, \nu(t) \equiv 0$ and, analogously, the mode $\nu(t)$ must provide a certain force only in the rhs of Eq.(33) when $\mu(t) \equiv 0$ and $\gamma(t) \equiv 0$. These properties can be easily seen from Eqs.(33 36). One can also reveal that the mode $\mu(t)$, whose symmetry is higher than those of the modes $\nu(t)$ and $\gamma(t)$, does not produce any forces in the rhs of Eqs.(34) and (35), if $\nu(t) \equiv 0$ and $\gamma(t) \equiv 0$. Similarly, one can verify that the modes $\nu(t)$ and $\mu(t)$ do not produce any forces in the rhs of Eq.(35) when $\mu(t) \not \equiv 0, \nu(t) \not \equiv 0$, but $\gamma(t) \equiv 0$.

Thus, we confirmed the typical bush structure of the dynamical equations 33 35 in terms of the individual modes.

\section{$5 \quad$ Stability of bushes of vibrational modes}

All possible bushes of vibrational modes for the octahedral structure depicted in Fig. [ were found by means of group-theoretical methods in the previous section, and these results 
are independent of the type of interactions between individual particles of our mechanical system. On the other hand, studying the stability of bushes of modes depends essentially on the concrete type of interactions in the considered system, and we suppose that they can be described by the Lennard-Jones potential (21). Then the bush stability can be analyzed with the aid of numerical methods.

\subsection{Setting up the problem}

First of all, we must define what we mean speaking about stability of bushes of vibrational modes, since the term "stability" is used in very different senses. Let us excite a given bush $B\left[G_{D}\right]$ with the aid of the appropriate initial conditions (see below). We can speak about the energy $E_{0}$ of the mechanical system in this initial state. Remember that the given bush is a closed dynamical object, i.e. energy $E_{0}$ can spread only among its own modes. Let us decompose the $3 \times N$-dimensional vector $\mathbf{X}(t)$, describing our bush $B\left[G_{D}\right]$ at a certain instant $t$ into the complete set of orthonormal basis vectors from Table 3 . As a result, we obtain a definite collection of nonzero coefficients $\mu_{i}^{(j)}(t)$ from Eq.(2). We can repeat this procedure at other times during the evolution of the system, thereby obtaining the time evolution of $\mu_{i}^{(j)}(t)$. During this time evolution, $\mu_{i}^{(j)}(t)$ may pass through zero but will never be identically equal to zero. This set of modes forms the excited bush $B\left[G_{D}\right]$ of vibrational modes.

Increasing gradually the energy $E_{0}$ of the initial excitation, we can detect the loss of stability of the bush $B\left[G_{D}\right]$. Indeed, beyond a certain value of $E_{0}$, after some transient time interval, the complete set of modes with nonzero amplitudes $\mu_{i}^{(j)}(t)$ becomes larger than that of the bush $B\left[G_{D}\right]$. Obviously, this phenomenon leads to the appearance of a certain new bush $\tilde{B}\left[\widetilde{G_{D}}\right]$ which includes the old bush $B\left[G_{D}\right]$ and whose symmetry $\widetilde{G_{D}}$ is lower than the symmetry group $G_{D}$ of the bush $B\left[G_{D}\right]\left(\widetilde{G_{D}} \subset G_{D}\right)$, because all nonzero vibrational modes with symmetry higher than or equal to $G_{D}$ are already contained in $B\left[G_{D}\right]$.

Thus, the loss of stability of a given bush is accompanied by the spontaneous breaking of symmetry of the initial excited dynamical regime, described by this bush. We already discussed this phenomenon in Sec.2 and concluded that its cause is analogous to that of parametric resonance.

We can also say this in other words. A given bush $B\left[G_{D}\right]$ represents a certain dynamical regime in the considered mechanical system. Its modes interact with other modes which do not belong to $B\left[G_{D}\right]$, but these interactions must be of parametric (not force!) type only (see Sec.2.2). For the appropriate initial conditions we can get into a region of unstable movement. As a result, some new modes are excited which were forbidden by the principle of determinism of classical mechanics. Then we can speak about the loss of stability of the original bush $B\left[G_{D}\right]$ and its transformation into a larger bush $\tilde{B}\left[\widetilde{G_{D}}\right]$ with $\widetilde{G_{D}} \subset G_{D}$.

It was found from numerical experiments that the boundaries of stable (unstable) domains for bushes of vibrational modes depend not only on the initial energy $E_{0}$, but on the way of excitation, as well. Because of this reason, we will fix the way of initial excitation.15

Let us first discuss the loss of stability of the bushes from Table 11. The root mode of each of these bushes is determined by a single time-dependent coefficient $\mu_{i}^{(j)}(t)$ whose initial value at $t=t_{0}$ we will denote by the symbol $\mu_{0}\left(\mu_{0} \equiv \mu_{i}^{(j)}\left(t_{0}\right)\right)$. At the initial instant $t=t_{0}$, we fix the coordinates of all particles of the mechanical system in such a way that their displacements correspond to the appropriate root mode with amplitude $\mu_{0}$, while their

\footnotetext{
${ }^{15}$ Similar results can be also obtained for other ways of excitation.
} 
velocities are equal to zero. Namely this choice of initial conditions determines the above mentioned way of excitation of a given bush.

Using these initial conditions we solve numerically the exact dynamical equations of the considered mechanical system with 18 degrees of freedom, and analyze the set of nonzero modes $\mu_{i}^{(j)}(t)$ in the decomposition (2) of the vector $\mathbf{X}(t)$ obtained as a result of this solving. Then we gradually increase the value $\mu_{0}$ and repeat the procedure just described until the number of nonzero modes $\mu_{i}^{(j)}(t)$, at some value $\mu_{0}=R$, becomes larger than that of the bush $B\left[G_{D}\right]$. We will refer to $R$ as the threshold of stability of the given bush. Obviously, in such a way we obtain the upper boundary of the first stability region of $B\left[G_{D}\right]$ in the one-dimensional space of all possible values $\mu_{0}\left(0<\mu_{0} \leq R\right) .16$

The threshold values $R$, with the appropriate numerical accuracy, 17 for different bushes for the octahedral structure without a centered particle can be found in the last column of Table 1. For example, the threshold $R=0.028$ corresponds to bush $B 3\left[D_{2 d}^{\prime}\right]$. It means that below this threshold only three nonzero modes can be revealed in the decomposition of the appropriate vector $\mathbf{X}(t): \mu_{3}^{(8)}(t)$ (root mode) and $\mu_{1}^{(1)}(t), \mu_{1}^{(5)}(t)$ (secondary modes), while beyond this value of $R$ some new modes $\mu_{i}^{(j)}(t)$ appear in the decomposition (2) of the vector $\mathbf{X}(t)$. We treat this fact as evidence of the loss of stability of bush $B 3\left[D_{2 d}^{\prime}\right]$ and its transformation to a new bush of higher dimension. Note that in the present paper, we do not determine what this new bush is, because realization of such a goal requires a sufficient time due to some computational errors problems.

We already discussed the bush $B 6\left[C_{2 v}^{\prime}\right]$ from Table 1 which can be excited either by an initial excitation of the root mode $\varphi_{1}^{(8)}+\boldsymbol{\varphi}_{2}^{(8)}$ of the irrep $\Gamma_{8}$ with the amplitude $\mu_{1}^{(8)}(t)$, or by excitation of the root mode $\varphi_{1}^{(10)}-\varphi_{2}^{(10)}$ of the irrep $\Gamma_{10}$ with the amplitude $\mu_{1}^{(10)}(t)$. In such cases, we find two different threshold values $R_{1}$ and $R_{2}$ corresponding to the two different root modes.

A more complicated computational problem arises when we analyze the domains of stability for some bushes of modes from Table 2. Indeed, the root modes of bushes $B 10\left[D_{2 h}\right]$, $B 11\left[D_{2}^{\prime}\right], B 12\left[C_{2 v}\right], B 13\left[C_{2 v}^{\prime \prime}\right], B 14\left[C_{2}^{\prime}\right], B 16\left[C_{s}^{\prime}\right], B 17\left[C_{3}\right]$, include two independent functions $\mu_{i}^{(j)}(t)$. For some of the above bushes these functions are associated with basis vectors of one and the same irrep; in other cases they are associated with basis vectors of two different irreps. Therefore, we must now study the boundary of the stability domain in a two-dimensional space of initial values, say, $\mu_{1}\left(t_{0}\right)$ and $\mu_{2}\left(t_{0}\right)$, of the two appropriate time-dependent variables $\mu_{i}^{(j)}(t)$. Since studying of the form of such a boundary, by means of numerical experiments, requires a lot of computational time, we restrict ourselves to the determination of only the radius $R^{\prime}$ of a circle which with assurance lies in the stability domain. Namely these radii we give in the last column of Table 2 as certain estimation characteristics of stability domains of the appropriate bushes of vibrational modes.

In Table 2, as well as in Table 1, we encounter the case where a given bush can be excited by using different choices of the root mode, but now each of these modes is determined by two amplitudes $\mu_{i}^{(j)}(t)$. This is the bush $B 15\left[C_{s}\right]$ whose root modes are $\mu_{1}^{(8)}(t) \boldsymbol{\varphi}_{1}^{(8)}+\mu_{2}^{(8)}(t) \boldsymbol{\varphi}_{2}^{(8)}$ or $\mu_{1}^{(10)}(t) \boldsymbol{\varphi}_{1}^{(10)}+\mu_{2}^{(10)}(t) \boldsymbol{\varphi}_{2}^{(10)}$. Because of this reason, two different radii $R_{1}^{\prime}$ and $R_{2}^{\prime}$ are given in Table 8 corresponded to the above two variants of the root mode.

Finally, the root mode of the last bush $B 18\left[C_{i}\right]$ in Table 2 depends on three functions $\mu_{1}^{(7)}(t), \mu_{2}^{(7)}(t), \mu_{3}^{(7)}(t)$ and, therefore, we must study, in this case, the boundary of the stability

\footnotetext{
${ }^{16}$ Note that we do not study the other possible regions of stability of the given bush $B\left[G_{D}\right]$ in the present paper.

${ }^{17}$ We hope that numerical errors do not exceed unity in the last digits of numbers in our tables.
} 
domain in three-dimensional space. We give for this bush the radius $R^{\prime \prime}$ of a sphere which with certainty belongs to the stability domain.

\subsection{Numerical results on bush stability for octahedral structure without centered particle}

Up to this point our consideration was based on group-theoretical methods which are independent of the type of interactions between particles in the mechanical system. Now we intend to discuss the findings on bush stability using for this purpose the concrete type of interactions described by the Lennard-Jones potential already discussed in Sec. 1 (see Eq. (21)).

First of all, we must examine the stability of the octahedral structure depicted in Fig.1 in the equilibrium state. To this end, we found squares of all eigenfrequencies in the harmonic approximation with the aid of the MAPLE package and made their classification in accordance with the irreps of the group $O_{h}$. We obtained the following results:

$$
\begin{array}{cc}
\omega^{2}\left[\Gamma_{1}\right]=61.082 ; & \omega^{2}\left[\Gamma_{5}\right]=13.835 ; \\
\omega^{2}\left[\Gamma_{7}\right]=31.498 ; & \omega^{2}\left[\Gamma_{8}\right]=15.346 ; \\
\omega^{2}\left[\Gamma_{9}\right]=0 ; & \omega^{2}\left[\Gamma_{10}\right]=46.843 ; 0 .
\end{array}
$$

Here $\omega^{2}\left[\Gamma_{j}\right]$ is a square of the frequency corresponding to the irrep $\Gamma_{j}$.

The irreps $\Gamma_{2}, \Gamma_{3}, \Gamma_{4}$ and $\Gamma_{6}$ are not contained in the decomposition of the full vibrational representation $\Gamma_{\text {mech }}$ of the octahedral structure and, as a consequence, there are no vibrational frequencies corresponding to them.

Note, $\omega^{2}\left[\Gamma_{9}\right]$ and the last value of $\omega^{2}\left[\Gamma_{10}\right]$ are equal to zero. They correspond, respectively, to pure rotation and pure translation of the mechanical system as a whole, and must be excluded from the further discussion because we consider vibrational regimes only.18 All other $\omega^{2}\left[\Gamma_{j}\right]$ are positive and this proves that the equilibrium state of our mechanical system is stable.

Let us also write down the value $a_{0}$ of the edge of the regular octahedron depicted in Fig. [1 in equilibrium:

$$
a_{0}=1.117 \text {. }
$$

Hereafter, we use such dimensionless units that $A=1$ and $B=1$ in the formula (21) for the Lennard-Jones potential (see Sec. 4).

Now we consider our results on the stability of dynamical regimes described by bushes of vibrational modes. The computational scheme, outlined in the previous section, and fourth order Runge-Kutta method were used for appropriate numerical experiments. The threshold values $R, R^{\prime}, R^{\prime \prime}$ characterizing the size of stability domains for all bushes are given in the last column of Tables 1, 2. It can be seen from these tables that the size of stability domains differs very considerably for different bushes: $0.001 \leq R \leq 0.078,0.001 \leq R^{\prime}<0.027$.

The one-dimensional bush $B 1\left[O_{h}\right]$, consisting of the breathing mode only, possesses the minimal "stability reserve": $R=0.001$. Obviously, this is a very small value for $R$, because the displacements of particles corresponding to it are approximately equal to 0.0005, while the equilibrium edge $a_{0}$ of our octahedron is equal to 1.117 according to Eq.(39). Thus, the bush $B 1\left[O_{h}\right]$ exhibits very weak stability for the octahedral structure without a centered

\footnotetext{
${ }^{18}$ Remember that movements of rotation-vibration type were excluded from our consideration in the present paper.
} 
particle. Nevertheless, if a particle in the center of the octahedron depicted in Fig.11 is present, the threshold value $R$ for this bush can be up to 100-1000 times greater, depending on the force properties of the centered particle (see Sec. 5.4).

The maximal value $R$ corresponds to the bush $B 9\left[D_{3 d}\right](R=0.078)$, demonstrating how strong can be the difference between the stability properties of dynamical regimes with different character of displacements of individual particles (note that using Tables 1, 2, 3, we can imagine the character of movement of the mechanical system for any given bush).

Our main result can be formulated as follows: all bushes of vibrational modes possess stability domains of a finite size for the octahedral structure with the Lennard-Jones potential.

\subsection{Interactions between different modes}

In many physical papers, pair interactions between modes are discussed (compare the phonon-phonon interactions in the presence of anharmonic terms in the appropriate Hamiltonian). In a rigorous sense, such a setting of the problem is incorrect and can be used only as a first step of investigation, for example, to obtain some estimations. Indeed, we can formally write the Lagrange equations describing dynamics of only two arbitrarily chosen modes, but this new two-dimensional dynamical system may not correspond to any real dynamical regime in the original mechanical system.

The importance of the bush theory is brought about, above all, by the following consequence: choosing a given bush, we can guarantee that the full collection of its modes is closed, i.e. that the appropriate dynamical regime including only these modes can really exist in the considered mechanical system. As a consequence, the Lagrange equations, obtained for the complete set of the modes of a given bush, are exact.

Nevertheless, studying the interactions between pairs of different normal modes may be useful in certain cases as an approximate method for revealing some essential properties of nonlinear dynamics of the mechanical system. Let us illustrate this idea using the following examples.

1. We consider a possibility of the parametric excitation of "sleeping" modes19, belonging to the irreps $\Gamma_{5}, \Gamma_{7}, \Gamma_{8}$ and $\Gamma_{9}$, brought about by their pair interactions with the breathing mode $\mu_{1}^{(1)}(t)$. In other words, we must find the threshold values $\mu_{1}^{(1)}(0) \equiv \mu_{c}$ beyond which each of these previously sleeping modes can appear because of its interaction with the "active" mode $\mu_{1}^{(1)}(t) \neq 0$. The following results were obtained by means of numerical experiments:

$$
\begin{gathered}
\Gamma_{5}\left[\mu_{1}^{(5)}(t), \mu_{2}^{(5)}(t)-\mu_{c}=0.020\right], \\
\Gamma_{7}\left[\mu_{1}^{(7)}(t), \mu_{2}^{(7)}(t), \mu_{3}^{(7)}(t)-\mu_{c}=0.173\right], \\
\Gamma_{8}\left[\mu_{1}^{(8)}(t), \mu_{2}^{(8)}(t), \mu_{3}^{(8)}(t)-\mu_{c}=0.001\right], \\
\Gamma_{10}\left[\mu_{1}^{(10)}(t), \mu_{2}^{(10)}(t), \mu_{3}^{(10)}(t)-\mu_{c}=0.333\right] .
\end{gathered}
$$

Note that modes listed in square brackets next to the symbol of the appropriate irrep possess the identical threshold value $\mu_{c}$.

According to Eq.(40), the minimal threshold value $\mu_{c}$ corresponds to the modes of the irrep $\Gamma_{8}\left(\mu_{c}=0.001\right)$, and this value coincides with the threshold of stability $R=0.001$ of the one-dimensional bush $B 1\left[O_{h}\right]$ pointed out in Table 11. It is essential, that when we

\footnotetext{
${ }^{19}$ We mean by this term the modes which do not belong to the bush generated by a given root mode $\left(\mu_{1}^{(1)}(t)\right.$, in our case) and which were not excited at the initial instant $t=t_{0}$.
} 
find this value $R$ for $B 1\left[O_{h}\right]$ by means of the numerical procedure described in Sec.5.1, we cannot reveal the cause of such a small value of $R$. Now we can assert that the bush $B 1\left[O_{h}\right]$ loses its stability because of the interactions with the modes namely of the irrep $\Gamma_{8}$ and, therefore, this bush must transform into a certain bush, containing these modes, such as $B 3\left[D_{2 d}^{\prime}\right], B 6\left[C_{2 v}^{\prime}\right]$, etc., but not into such bushes as $B 2\left[D_{4 h}\right], B 4\left[C_{2 v}\right]$, etc.

2. Let us discuss the threshold value $R=0.009$ of the stability of the bush $B 2\left[D_{4 h}\right]$ (see Table 1) in terms of pair interactions between different modes. This bush contains two modes: the root mode $\mu_{1}^{(5)}(t)$ and the secondary mode $\mu_{1}^{(1)}(t)$. We can find the threshold of the initial value $\mu_{1}^{(5)}(0) \equiv \mu_{c}$ beyond which the active mode $\mu_{1}^{(5)}(t)$ excites, by means of its parametric action, other modes which were previously sleeping. (There is one and only one mode $-\mu_{1}^{(1)}(t)$ - which is brought into the vibrational process by force originating from the active mode $\mu_{1}^{(5)}(t)$.) We obtained the following results:

$$
\begin{gathered}
\Gamma_{5}\left[\mu_{2}^{(5)}(t)-\mu_{c}=0.273\right], \\
\Gamma_{7}\left[\mu_{1}^{(7)}(t), \mu_{2}^{(7)}(t)-\mu_{c}=0.560 ; \mu_{3}^{(7)}(t)-\mu_{c}=0.403\right], \\
\Gamma_{8}\left[\mu_{1}^{(8)}(t)-\mu_{c}=0.344 ; \mu_{2}^{(8)}(t)-\mu_{c}=0.344 ; \mu_{3}^{(8)}(t)-\mu_{c}=0.455\right], \\
\Gamma_{10}\left[\mu_{1}^{(10)}(t)-\mu_{c}=0.616 ; \mu_{2}^{(10)}(t)-\mu_{c}=0.616 ; \mu_{3}^{(10)}(t)-\mu_{c}=0.577\right] .
\end{gathered}
$$

Note that unlike the case where the breathing mode was active (see Eq. (40)), threshold values $\mu_{c}$ for the excitation of sleeping modes by the active mode $\mu_{1}^{(5)}(t)$ can be different for the different modes of one and the same multidimensional irrep.

A remarkable fact can be revealed in analyzing the above threshold values given in Eq.(41). Indeed, all $\mu_{c}$, corresponding to the initial value of the root mode $\mu_{1}^{(5)}(t)$, are essentially greater than the threshold $R=0.009$ for the loss of stability of the considered bush $B 2\left[D_{4 h}\right]$. On the other hand, as we already know, the threshold for the parametric excitation of the modes associated with the irrep $\Gamma_{8}$ by action from the active mode $\mu_{1}^{(1)}(t)$ is a very small value: $\mu_{c} \equiv \mu_{1}^{(1)}(0)=0.001$. This fact suggests that the sufficiently weak stability of the bush $B 2\left[D_{4 h}\right]$ brought about by the parametric excitation of some sleeping modes originated not from the root, but from the secondary mode of our bush! Indeed, if we consider dynamics of the bush $B 2\left[D_{4 h}\right]$ when $\mu_{1}^{(5)}(0)$ are slightly higher than 0.009 , the value of its secondary mode $\mu_{1}^{(1)}(t)$ does reach the threshold value 0.001 which can lead to excitation of the modes of the irreps $\Gamma_{8}$.

Thus, we encounter now the case where a given bush loses its stability because of the phenomenon similar to the parametric resonance induced by its secondary mode, and this property demonstrates, in particular, that a bush is an indivisible dynamical object.

\subsection{Stability of bushes of vibrational modes for octahedral struc- ture with the centered particle}

First of all, let us note that all octahedral molecules, known to us at the present time, possess an atom in the center of the octahedron (see Fig.(1). This fact suggests that the stability of such structures can be greater than that of the mechanical system considered in the previous sections. Taking into account this hint, we examined bush stability for the mechanical structure depicted in Fig.1, supposing that the particle in the center of the octahedron is described by the Lennard-Jones potential different from that of six peripheral particles. 
Let us assume that the Lennard-Jones potential of the form

$$
\widetilde{U}(r)=\frac{1}{r^{12}}-\frac{B}{r^{6}}
$$

with $B>1$ corresponds to the centered particle, while all other particles are described by the potential (42) with $B=1$. Such an assumption provides us a possibility to make the attractive part of the potential of the centered particle greater than that of peripheral atoms in spite of the same repulsive part.

We will consider only several bushes of modes for the centered structure to show that the size of the stability domain can be increased essentially by means of the appropriate choice of the value B. Note for the beginning of the discussion, that all bushes from Tables 1, 2 are relevant bushes for centered structure, as well as for that without the particle in the center of the octahedron, if this particle is fixed 20

Let us discuss some results of the appropriate numerical experiments. The boundary of the stability domain for the bush $B 1\left[O_{h}\right]$ can be increased from the threshold value $R=0.001$ (see Table 1) up to the value $R=1.01$ for the case of the centered structure with $B=5.5$. (It is interesting that the function $R(B)$ for this bush is not monotonic). The threshold values $R$ for other considered bushes also become larger for $B=5.5$ in comparison with those for $B=1$. For example, we obtained $R=0.011$ for bush $B 2\left[D_{4 h}\right]$ instead of $R=0.009$ and $R=0.118$ for bush $B 4\left[C_{4 v}\right]$ instead of $R=0.003$. Note that the dependence of the boundary $R$ of stability domains on the value $B$ is essentially different for different bushes.

We can conclude that the stability of bushes of vibrational modes for the centered structure can be increased, and for some bushes substantially increased, in comparison to that of the mechanical system without the centered particle on account of the appropriate choice of the constant $B$ in the Lennard-Jones potential (42).

\section{Summary}

The specific dynamical objects — bushes of normal modes - whose theory was developed in [1, 2, 3], are discussed in the present paper for octahedral mechanical systems with point masses. Being a certain collection of modes, a given bush possesses some symmetry group which is a subgroup of the symmetry group of the mechanical system in equilibrium. The bush can be excited under definite initial conditions, and the energy of the initial excitation turns out to be trapped in this bush.

We found that there exist 18 different by symmetry bushes of vibrational modes for the considered mechanical system. We examined the stability of all these bushes for the case where interactions between particles are described by the Lennard-Jones potential. The main results are listed in Tables 1 and 2 .

It was proved that all of the above mentioned bushes possess stability domains of finite size and, therefore, they really can be treated as certain physical objects.

\section{Appendix 1. The point group $O_{h}$ and its irreducible representations}

The group $O_{h}$ consists of 48 symmetry elements. We can obtain all these elements with the aid of the different products of a certain set of generators. The minimal number of generators

\footnotetext{
${ }^{20}$ If the centered particle can move, we obtain some additional bushes to those in Tables 1 , 2 .
} 
for group $O_{h}$ is equal 2, but it is more convenient, for our purpose, to use an extended set of generators. In notation of the handbook by Kovalev [9], we chose the following five generators: $h_{2}, h_{3}, h_{5}, h_{13}, h_{25}$. Here $h_{2}, h_{3}$ and $h_{13}$ are $180^{\circ}$ rotations about the two-fold axes [100], [010] and [110], respectively; $h_{5}$ is a $240^{\circ}$ rotation about the three-fold axis [111], and $h_{25}$ is the inversion. Using these generators we can obtain, step by step, the following chain of groups: $C_{2}\left[h_{2}\right] \rightarrow D_{2}\left[h_{2}, h_{3}\right] \rightarrow T\left[h_{2}, h_{3}, h_{5}\right] \rightarrow O\left[h_{2}, h_{3}, h_{5}, h_{13}\right] \rightarrow O_{h}$. Each group is obtained from the preceding group by adding one new generator.21

The group $O_{h}$ has ten irreducible representations $\Gamma_{j}(j=1, \ldots, 10)$. Matrices of the generators of this group for all irreps are given below in Table 4 .

Table 4: Matrices of the generators of the group $O_{h}$

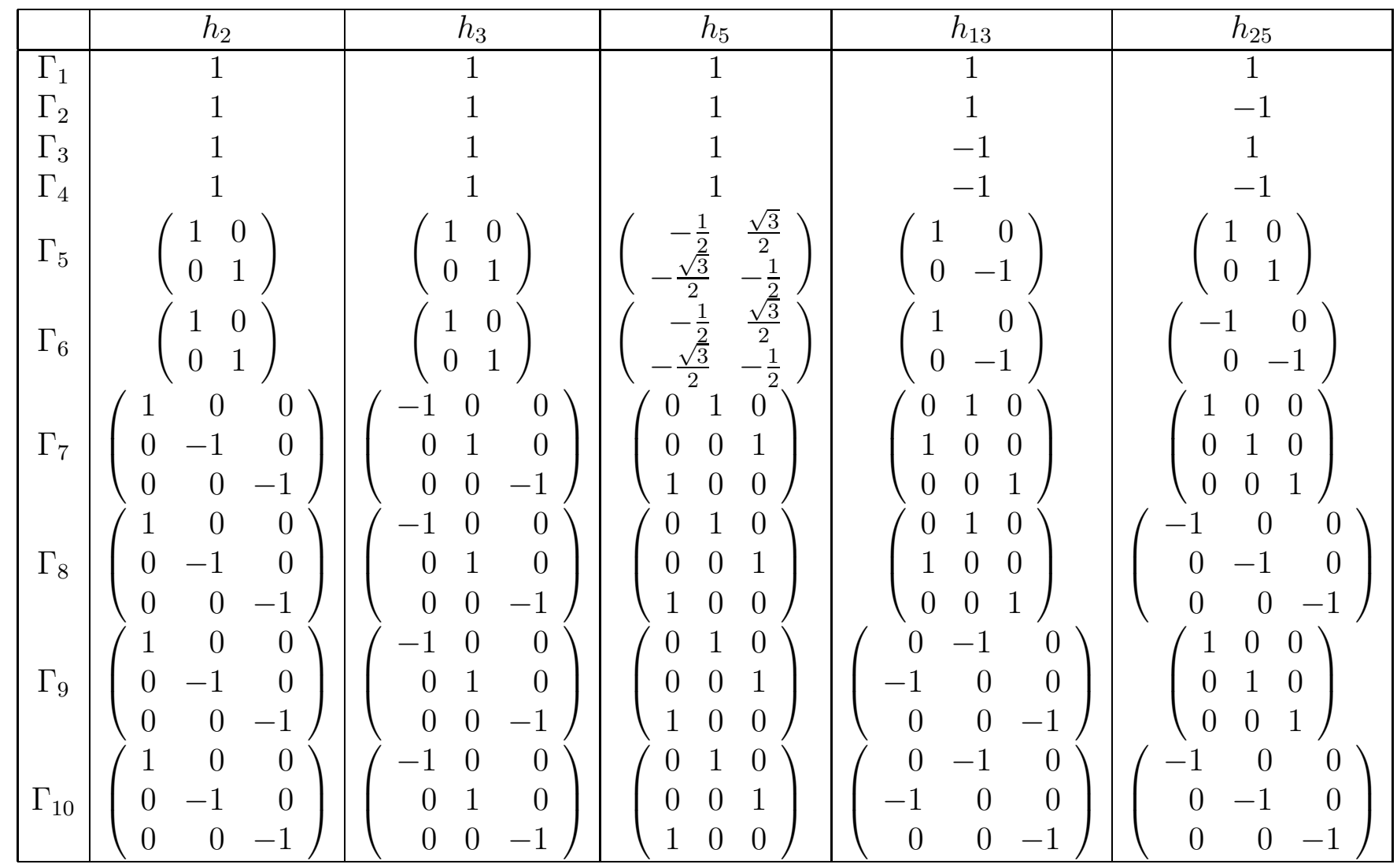

\section{Acknowledgements}

The authors thank Prof. V.P. Sakhnenko for useful discussions and Prof. H.T. Stokes for his great help in correction of the text of the present paper.

\section{References}

\footnotetext{
${ }^{21}$ We use Schoenflies symbols for the symmetry groups and point out their generators in square brackets next to these symbols.
} 
[1] V.P. Sakhnenko, G.M. Chechin, Symmetrical selection rules in nonlinear dynamics of atomic systems. Dokl. Akad. Nauk 330, 308 (1993). [Phys. Dokl. 38, 219 (1993)].

[2] V.P. Sakhnenko, G.M. Chechin, Bushes of modes and normal modes for nonlinear dynamical systems with discrete symmetry. Dokl. Akad. Nauk. 338, 42 (1994). [Phys. Dokl. 39, 625 (1994)].

[3] G.M. Chechin, V.P. Sakhnenko, Interactions between normal modes in nonlinear dynamical systems with discrete symmetry. Exact results. Physica D. 117, 43 (1998).

[4] G.M. Chechin, V.P. Sakhnenko, H.T. Stokes, A.D. Smith, D.M. Hatch, Non-linear normal modes for systems with discrete symmetry. Int. J. Non-Linear Mech. 35, 497 (2000).

[5] The software package, ISOTROPY, is available on the Internet at http://www.physics.byu.edu/] stokesh/isotropy.html .

[6] G.M. Chechin, Computers and group-theoretical methods for studying structural phase transition. Comput. Math. Applic. 17, 255 (1989).

[7] G.M. Chechin, V.P. Sakhnenko, M.Yu. Zekhtser, H.T. Stokes, S. Carter, D.M. Hatch, Bushes of normal modes for nonlinear mechanical systems with discrete symmetry. World Wide Web Proceedings of the 3rd ENOC conference http://www.midit.dtu.dk

[8] G.M. Chechin, N.V. Novikova, A.A. Abramenko, Bushes of vibrational modes for FermiPasta-Ulam chains. To be published.

[9] O.V. Kovalev, Representations of the Crystallographic Space Groups: Irreducible Representations, Induced Representations and Corepresentations. Gordon and Breach, Amsterdam, 1993.

[10] G.M. Chechin, V.A. Koptsik, Relation between multidimensional representations of the Fedorov groups and the groups of color symmetry. Comput. Math. Applic. 16 5-8 (1988) $521-536$ 
Figure 1: Octahedral mechanical system. 
This figure "octfin.gif" is available in "gif" format from: http://arxiv.org/ps/cond-mat/0112213v1 